\title{
The flowering plants and ferns of Anacapa Island, California
}

\author{
Steve JunaK ${ }^{1, *}$ and Ralph Philbrick ${ }^{2}$ \\ ${ }^{1}$ Santa Barbara Botanic Garden, 1212 Mission Canyon Road, Santa Barbara, CA 93105 \\ ${ }^{2}$ Deceased. 29 San Marcos Trout Club, Santa Barbara, CA 93105
}

\begin{abstract}
Aвstract.-The 3 islets of Anacapa Island, with a combined area of $1.1 \mathrm{mi}^{2}\left(2.9 \mathrm{~km}^{2}\right)$, lie $13 \mathrm{mi}(20 \mathrm{~km})$ off the coast of southern California. Historically, each of Anacapa's islets has been subjected to periodic grazing by sheep, and the eastern islet has also had a sizeable population of introduced rabbits. In spite of these past perturbations, the recovery of the island's vegetation has been remarkable since sheep removal in 1937. Despite its small size, Anacapa Island supports a surprising diversity of vascular plants, with nearly 200 native taxa from 50 plant families. Twenty-one of these native taxa are restricted to the California Islands; one annual species of Malacothrix occurs as a narrow endemic found only on Middle Anacapa Island, while another annual subspecies of Malacothrix is known only from West and East Anacapa. The floral diversity of this island has apparently been influenced by its close proximity to the mainland and other larger islands and by its diverse topography. Anacapa Island supports a slightly larger native flora than San Miguel Island, which is about 13 times larger and is located about $50 \mathrm{mi}(80 \mathrm{~km})$ to the west. The Anacapa native flora is more than twice the size of that found on Santa Barbara Island, which has almost the same area as Anacapa Island but is located approximately $62 \mathrm{mi}(100 \mathrm{~km})$ to the southeast. Even though botanical exploration on Anacapa began in 1889, the current work represents the first complete enumeration of the island's flora.
\end{abstract}

Resumen.-Los tres islotes de la isla Anacapa, con un área combinada de $1.1 \mathrm{mi}^{2}\left(2.9 \mathrm{~km}^{2}\right)$, se encuentran a 13 millas $(20 \mathrm{~km})$ de la costa del sur de California. Históricamente, cada uno de los islotes de Anacapa fue sometido a pastoreos de ovejas periódicos, y el islote oriental presentó, además, una población considerable de conejos introducidos. Pese a estas alteraciones pasadas, la recuperación de la vegetación de la isla fue notable desde la remoción de las ovejas en 1937. A pesar de su pequeño tamaño, la isla Anacapa tiene una sorprendente diversidad de plantas vasculares, con casi 200 taxa nativos de 50 familias de plantas. Veintiuno de estos taxa nativos se limitan a las Islas de California; una especie anual de Malacothrix es endémica y limitada al centro de la isla de Anacapa, mientras que otra subespecie anual de Malacothrix se conoce solo al oeste y al este de Anacapa. La diversidad floral de esta isla estuvo aparentemente condicionada por su proximidad al continente y a otras islas de mayor tamaño, y por su diversa topografía. La isla Anacapa consta de una flora nativa un poco más grande que la de la isla San Miguel que es, aproximadamente, 13 veces más grande y que se encuentra a unas $50 \mathrm{mi}(80 \mathrm{~km})$ al oeste. La flora nativa de Anacapa doblega el tamaño de la que se encuentra en la isla Santa Bárbara, que tiene casi la misma superficie que Anacapa, pero que se ubica, aproximadamente, a $62 \mathrm{mi}(100 \mathrm{~km})$ al sureste. Aunque la exploración botánica comenzó en Anacapa en 1889, el trabajo actual representa el primer inventario completo de la flora en la isla.

On a clear day several of the 8 California Channel Islands can be seen from Los Angeles, one of the largest metropolitan areas in the United States. The 8 islands have been divided into 2 major groups: (1) the Northern Channel Islands of San Miguel, Santa Rosa, Santa Cruz, and Anacapa, and (2) the Southern Channel Islands of Santa Barbara, San Nicolas, Santa Catalina, and San Clemente. These islands represent emergent portions of a complex system of submarine canyons and ridges in a geomorphic province referred to as the California Continental Borderland (Vedder 1976).

\section{STUdY Site}

Anacapa Island is the smallest, easternmost portion of the Northern Channel Islands. Its closest neighbor in the island chain is Santa Cruz, which is located $5 \mathrm{mi}(8 \mathrm{~km})$ to the west. On California's mainland coast, Port Hueneme is the closest point at $13 \mathrm{mi}(20 \mathrm{~km})$ to the northeast. Anacapa is located in Ventura County and is part of Channel Islands National Park.

Roughly $5 \mathrm{mi}(8 \mathrm{~km})$ long and with a maximum width of about $0.7 \mathrm{mi}(1 \mathrm{~km})$, Anacapa is a long, narrow chain composed of 3 islets

*Corresponding author: sjunak@sbbg.org 
running along a west-east axis. This orientation creates an onshore side facing mainland California to the north and an offshore side facing the open Pacific Ocean to the south. The 3 islets named West, Middle, and East Anacapa have a combined total area of about $1.1 \mathrm{mi}^{2}\left(2.9 \mathrm{~km}^{2}\right)$ and are surrounded by precipitous rocky cliffs, most of which drop off directly into the sea. Gleason (1950) provided a marvelous description of these cliffs:

The perpendicular sides emerge from deep water, the lava formation hanging like tattered garments. The sea has honey-combed the bases into caves which echo to the sound of seal and sea lion.... The heaving ground swell enters some of the lower caves and air pressure thus created sends out a blast of spray accompanied by a startling hiss and snort.

West Anacapa, the largest of the 3 islets (approximately 449 acres or $181.8 \mathrm{ha}$ ), has the most topographic diversity and 2 major peaks (acreage estimates for all islets from Roberts 1979). Summit Peak measures $930 \mathrm{ft}$. $(283.5$ $\mathrm{m})$ and Camel Peak reaches $811 \mathrm{ft} .(247.2 \mathrm{~m})$ above sea level. The offshore side of West Anacapa is extremely steep and offers a sheer wall of rock to the sea. The relatively gentle slopes on the onshore side, however, include several north-south-running canyons with luxuriant vegetation.

Middle and East Anacapa are basically flattopped mesas surrounded by nearly vertical sea cliffs which reach heights of over $200 \mathrm{ft}$. $(60 \mathrm{~m})$. Middle Anacapa reaches a maximum elevation of $325 \mathrm{ft}$. (99.1 m) and has an area of about 175 acres (70.8 ha). East Anacapa, with its highest point at $240 \mathrm{ft}$. $(73.2 \mathrm{~m})$ above sea level, has an area of about 106 acres (43 ha).

Geologically, Anacapa is almost entirely composed of dark-colored Miocene-age Conejo Volcanics with some modest-to-significant interbeds of lighter-colored San Onofre Breccia, also of Miocene age (Scholl 1960, Johnson 1979).

Anacapa Island essentially has a Mediterranean climate with mild, frost-free, wet winters and dry, warm summers. Coastal fog can be persistent in the summer months, however, and can significantly affect maximum and minimum temperatures. Climatological data for East Anacapa are sporadic and limited, and data are lacking for both West and Middle Anacapa. Dunkle (1950) reported an average annual rainfall for East Anacapa of 12.58 inches
$(31.95 \mathrm{~cm})$ for an unspecified 8-year period. He added that "this period includes two rather exceptional wet years." Only sporadic precipitation data are available for the years 1946-1968; the average annual figure for an 11-year period (1947, 1951-1952, 1960-1967) was 6.59 inches $(16.74 \mathrm{~cm}$ ) (Weissman and Rentz 1977). Precipitation measured on East Anacapa by the National Park Service averaged 7.55 inches $(20.45 \mathrm{~cm})$ annually across 9 calendar years between 2008 and 2016 (Western Regional Climate Center 2017). In contrast, the Stanton Ranch at approximately the same elevation on Santa Cruz Island received an average annual rainfall of 19.71 in (50.06 $\mathrm{cm}$ ) for an 89-year period (1904-1993) (Junak et al. 1995). Dunkle, Weissman, and Rentz attributed the low rainfall figures for East Anacapa to a rain shadow created by Santa Cruz Island and West Anacapa. It is clear that more data are needed to more completely understand Anacapa's climate.

Surface flow of freshwater is lacking on Middle and East Anacapa, except immediately after rain events. The only reliable source of freshwater on the island is in a large cave near sea level on the north side of West Anacapa. Yates (1890) reported a flow of 70 gallons per day from this cave spring, which apparently was used by Native Americans as a water source.

The history of human land use on Anacapa Island since the mid-1800s has been documented by Roberts $(1979,1983)$ and Livingston (2006). Sheep were reportedly present on Anacapa as early as 1869 , but most were removed by 1937. Middle Anacapa was used as the headquarters for sheep ranching activities, but sheep were present on all islets for varying amounts of time. Rabbits were introduced to East Anacapa in the 1930s or 1940s (Paul Collins, personal communication, 2016; McEachern et al. 2016) and were still present there in the 1960s (Banks 1966).

\section{History of Botanical ExPLORATION}

Botanical exploration on Anacapa Island began in the late 1880s and early 1890s with the visits of Lorenzo Gordin Yates, an Englishborn dentist, naturalist, and author who moved to Santa Barbara in 1882 (Camp 1963). He was the president of the Santa Barbara Society of Natural History and had a wide variety of 
interests, including anthropology, botany, conchology, mineralogy, paleontology, and zoology. Yates landed on Middle Anacapa in 1889 with a group of artists and scientists and collected botanical specimens on the island in the early 1890s (his collections are labeled with the date "about 1893").

Subsequent botanical collections were made on the island by the following individuals (names of collectors are followed by the year or years when collections were made by that person):

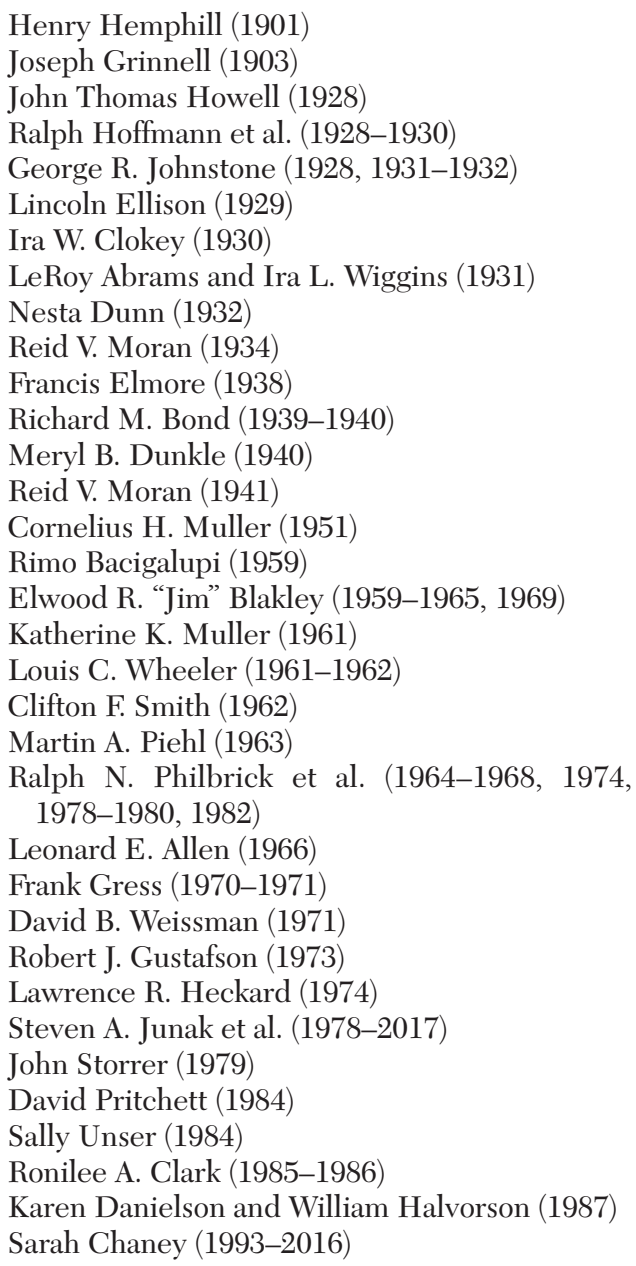

Our knowledge of Anacapa's flora, both past and present, is the result of the collective efforts of these scientific collectors and observers. Unfortunately not much was collected there in the early 1900s; there was a 25 -year gap in botanical documentation efforts (between 1903 and 1928) during the sheep ranching era on the island. There was another gap of about 18 years (between 1941 and 1959), starting in the World War II years, when the islands were closed to yachts and travel across the Santa Barbara Channel was rigidly controlled by defense authorities (Junak et al. 1995). Although Cornelius Muller visited the island in 1951, he only collected a few specimens on that trip. As was the case with most of the Channel Islands, grazing animals were turned loose and caused significant ecological disturbance before botanical collectors had the chance to document the native flora.

\section{FLORA OF ANACAPA}

The vascular flora of Anacapa Island consists of 196 native taxa, including 2 taxa that are only known from Anacapa and 21 additional taxa that are endemic to 2 or more of the California Islands (itemized in Appendix). In terms of life form, the insular endemics include 1 tree, 14 shrubs or subshrubs, 1 herbaceous perennial, 1 perennial vine, and 6 annual herbs. As to distribution on other islands, 9 of the insular endemics are found on 1 or more of the other Northern Channel Islands (in addition to Anacapa), 11 are shared with the Northern and Southern Channel Islands, and 1 is shared only with the Southern Channel Islands.

In terms of distribution on the islets of Anacapa, 170 of the native taxa have been documented on West Anacapa, 129 are known to occur on Middle Anacapa, and 87 have been collected on East Anacapa. A total of 19 of the insular endemics known from the island occur (or have occurred) on West Anacapa, 14 are found on Middle Anacapa, and 9 are known from East Anacapa.

The floral diversity of Anacapa has apparently been influenced by its close proximity to the mainland and other larger islands and by its diverse topography. Anacapa Island supports a slightly larger native flora than San Miguel Island, which is about 13 times larger and located about $80 \mathrm{~km}$ to the west. The Anacapa native flora is more than twice the size of that found on Santa Barbara Island, which has almost the same area as Anacapa but is located approximately $100 \mathrm{~km}$ to the southeast (Junak 2008).

A total of 87 nonnative plant taxa have been documented on Anacapa Island (itemized in the Annotated Catalog of the Vascular Plants 
of Anacapa Island, see below). One additional cultivated plant (Amaryllis belladonna L.) was observed near buildings on East Anacapa in the early 1980s, but no specimen was collected to document its occurrence. Twentyone percent of the documented nonnative taxa were first found on West Anacapa, 22\% were first found on Middle Anacapa (especially in areas disturbed by nesting seagulls), and 34\% were first found on East Anacapa (especially along trails and in or near the campground). For the other $23 \%$ of the nonnative taxa found on Anacapa, the islet where the first collection was made was not specified by the collector.

Of the 87 nonnative plant taxa known on Anacapa, 1 was first collected on the island between 1900 and 1919, 20 were collected between 1920 and 1939, 10 between 1940 and 1959, 25 between 1960 and 1979, and 25 between 1980 and 1999. A total of 6 additional nonnative taxa have been documented since 2000. In other words, 56 (nearly 63\%) of the nonnative plant taxa known on Anacapa were encountered for the first time after 1960. This is not surprising because visitation by daytime users and overnight campers has increased dramatically during the last 50-60 years.

In recent years sources of invasive plant introductions on Anacapa have included nesting Western Gulls (Larus occidentalis) on Middle Anacapa (which disturb the habitat around their nests and bring items from California mainland dumps) and human visitors on East Anacapa (some of whom do not carefully clean their clothing, shoes, and equipment before coming to the island). Several new weeds were also found in imported gravel and soil on East Anacapa (which apparently contained seeds of those weedy plants).

\section{Annotated Catalog of the Vascular PlantS OF ANACAPA ISLAND}

Plants listed in this table are arranged alphabetically by family within 5 major plant groups (lycophytes, ferns, gymnosperms, eudicots, and monocots). Taxa presumed to be introduced to California and/or Anacapa Island by human activities are preceded by an asterisk (*). This list does not include plant taxa that have been planted near the buildings on the island unless they have survived without cultivation.
For most taxa, classification, nomenclature, and abbreviations of author names follow Baldwin et al. (2012). A few names were updated in accordance with the Jepson eFlora. Selected synonyms are shown in brackets (i.e., [ ]). Common names are mostly according to Abrams (1940-1951), Abrams and Ferris (1960), Junak et al. (1995), Junak et al. (1997), Smith (1998), and Baldwin et al. (2012).

Abundance ratings (rare, scarce, occasional, common, and abundant) and distribution descriptions are based on observations of the authors. Dates of first known collection from Anacapa Island are included for nonnative taxa.

Voucher specimens are cited for each taxon. These are arranged by islet (i.e., WA for West Anacapa, MA for Middle Anacapa, and EA for East Anacapa). Labels on some early voucher specimens did not include precise locality information; these are cited as A (for Anacapa Island). Label data cited have been edited in some cases (e.g., Frenchman's Cove on West Anacapa is consistently cited as Frenchy's Cove). Specimens without a collector's number are listed as "s.n."; herbarium accession numbers are cited if there is no collector's number. Abbreviations for herbaria where voucher specimens are deposited are those used in Holmgren et al. (1990). If no depository is cited, specimens can be found at the Santa Barbara Botanic Garden (SBBG). No effort was made to provide an exhaustive list of herbaria where particular voucher specimens have been deposited.

\section{LYCOPHYTES \\ SELAGINELLACEAE (Spike-Moss Family)}

Selaginella bigelovii Underw. bushy spike-moss Rare; ridgetops on West Anacapa.

WA, 18 May 1967, Philbrick \& Ricker B67-13.

\section{FERNS}

DRYOPTERACEAE (Wood Fern Family)

Dryopteris arguta (Kaulf.) Maxon wood fern Rare; vicinity of Cherry Canyon on West Anacapa and an unspecified location on Middle Anacapa.

WA, 9 May 1963, Blakley 5765 (SBBG, SD, UCR); MA, Sep 1966, Baker \& Baker s.n. (SBBG 55190). PolyPODIACEAE
(Polypody Family)

Polypodium californicum Kaulf. California polypody Scarce; north-facing slopes on all islets. 
WA, 30 Mar 1995, Junak WA-380 (RSA, SBBG); MA 2 Jun 1978, Philbrick \& Hochberg B78-300 (RSA, SBBG); EA, 26 Apr 1959, Blakley 2801.

\section{PTERIDACEAE}

(Maiden Hair Family)

Adiantum capillus-veneris L. southern maidenhair Rare; north-facing slopes in Box and Willow canyons on West Anacapa.

WA, 8 May 1980, Junak et al. WA-153.

Adiantum jordanii Mull. Hal. California maidenhair Scarce; north-facing slopes and canyons on West and Middle Anacapa.

WA, 18 May 1961, Blakley 4344 (RSA, SBBG, SD, UCR); MA, 1 May 1986, Junak MA-184 (CAS, SBBG).

Pellaea andromedifolia (Kaulf.) Fee coffee fern Scarce; north-facing slopes on West and Middle Anacapa.

WA, 6 Sep 1964, Philbrick \& Flinck B64-323; MA, 2 Jun 1978, Philbrick B78-312.

Pentagramma triangularis (Kaulf.) Yatsk. et al. subsp. triangularis [Pityrogramma $t$. (Kaulf.) Maxon var. $t$. ] goldback fern Scarce; north-facing slopes and canyons on West and Middle Anacapa.

WA, 28 Mar 1979, Junak et al. WA-45; MA, 2 Jun 1978, Philbrick \& Hochberg B78-297.

\section{GYMNOSPERMS}

CUPRESSACEAE

(Cypress Family)

*Hesperocyparis macrocarpa (Hartw.) Bartel [Cupressus $m$. Hartw.] Monterey cypress Rare; planted by buildings on East Anacapa as early as 1936 (Lighthouse Keeper logs at National Archives). Not seen recently.

EA, 25 Apr 1959, Blakley 2782.

\section{EUDICOTS \\ AIZOACEAE \\ (Iceplant Family)}

*Aptenia cordifolia (L.f.) Schwantes baby sun-rose Rare; disturbed flats near buildings on East Anacapa. First collected there in August 1974.

EA, 5 Aug 1974, Philbrick B74-141.

*Carpobrotus chilensis (Molina) N.E.Br. [C. aequilaterus (Haw.) N.E.Br., misapplied] sea-fig Scarce; flats and slopes near Frenchy's Cove on West Anacapa and near west end of East Anacapa. First collected on "beach" (islet not specified) in September 1930.

A, 22 Sep 1930, Hoffmann s.n. (SBBG 80047); WA, 18 May 1961, Muller 1176; EA, 26 Apr 1959, Blakley 2812.

*Carpobrotus edulis (L. ) N.E.Br. freeway iceplant Rare; disturbed flats near buildings on East Anacapa. First collected there in April 1979.

EA, 30 Apr 1979, Junak EA-19.
*Malephora crocea (Jacq.) Schwantes Common; disturbed flats and offshore bluffs on East Anacapa and in the eastern portion of Middle Anacapa. First collected at "west end of flat part" of East Anacapa in 1959. This taxon was abundant on East Anacapa, but has now been almost eradicated from the flats there; it is still persisting and slowly spreading on the offshore bluffs of Middle and East Anacapa, and is occasional on the terrace of Middle Anacapa.

MA, 5 May 1998, Junak MA-397; EA, 26 Apr 1959, Blakley 2813.

*Mesembryanthemum crystallinum L. crystalline iceplant Common; widespread on disturbed flats and slopes on all islets. First collected on the island (exact location and islet not specified) in June 1930.

A, 16 Jun 1930, Hoffmann s.n. (SBBG 77688); WA, 18 May 1961, Blakley 4359; MA, 28 Oct 1979, Junak et al. MA-41; EA, 24 Apr 1959, Blakley 2766a (CAS, SBBG).

*Mesembryanthemum nodiflorum L. small-flowered iceplant Common; widespread on disturbed flats and slopes on all islets. First collected on "bare ground at border of cliff" (exact location and islet not specified) in March 1928.

A, 11 Mar 1928, Hoffmann s.n. (SBBG 77689); WA, 18 May 1961, Blakley 4358 (CAS, RSA, SBBG); MA, 18 May 1961, Muller 1181; EA, 26 Apr 1959, Blakley 2788 (SBBG, US).

*Tetragonia tetragonioides L. New Zealand spinach Rare; known only from back beach area at Frenchy's Cove on West Anacapa. First collected there in May 1961.

WA, 27 May 1961, Wheeler 7806 (RSA, SBBG); WA, 31 Mar 1962, Blakley 4935 (RSA, SBBG); WA, 16 Nov 1969, Blakley 7022.

\section{AMARANTHACEAE \\ (Amaranth Family)}

*Amaranthus albus L. tumbleweed Rare; known from a single collection on flats near Sheep Camp landing on Middle Anacapa in June 1978. MA, 3 Jun 1978, Philbrick \& Hochberg B78-335.

Amaranthus powellii S. Watson Powell's amaranth Rare; known from a single collection on slopes below lighthouse on East Anacapa.

EA, 19 Aug 1988, Junak EA-90 (SBBG, UCR).

\section{ANACARDIACEAE (Sumac Family)}

Rhus integrifolia (Nutt. In Torr. \& A. Gray) Brewer $\&$ S. Watson lemonade berry Occasional; flats and steep coastal slopes on all islets.

WA, 18 May 1961, Blakley 4354 (CAS, JEPS, RSA, SBBG); MA, 7 Apr 1968, Philbrick \& Osborne B68-167; EA, 26 Apr 1959, Blakley 2807. 
Toxicodendron diversilobum (Torr. \& A. Gray) Greene poison-oak Scarce; canyons on north side of West Anacapa.

WA, 18 May 1961, Blakley 4381 (RSA, SBBG).

\section{APIACEAE \\ (Carrot or Celery Family)}

Apiastrum angustifolium wild celery Nutt. Scarce; moist slopes and flats on West and Middle Anacapa.

WA, 23 Apr 1982, Junak WA-189; MA, 1 Jun 1978, Philbrick \& Hochberg B78-272.

Daucus pusillus Michx. rattlesnake weed Occasional; flats on all islets.

WA, 9 May 1963, Piehl 63-190; MA, 4 May 1998, Junak MA-374; EA, 26 Apr 1959, Blakley 2814.

Sanicula arguta Greene ex J.M. Coult. \& Rose sharp-toothed snakeroot Occasional; moist flats and slopes on all islets.

WA, 18 May 1961, Blakley 4374; MA, 31 Mar 1962, Blakley 4895; EA, 27 Mar 1979, Junak et al. EA-2 (JEPS, SBBG).

\section{Asteraceae \\ (Sunflower Family)}

Achillea millefolium L. yarrow Occasional; flats and slopes on all islets.

WA, 18 May 1961, Blakley 4402 (CAS, SBBG); MA, 1 Apr 1984, Junak MA-274 (RSA, SBBG); EA, 25 Apr 1959, Blakley 2778 (CAS, JEPS, RSA, SBBG, SD).

Amblyopappus pusillus Hook. \& Arn. pineapple weed Common; open flats along terrace edges on all islets.

WA, 19 May 1928, Howell 3815 (CAS); MA, 9 May 1963, Blakley 5722 (CAS, RSA, SBBG); EA, 28 Apr 1993, Junak EA-116 (RSA, SBBG).

*Ambrosia acanthicarpa Hook. annual bur-sage Rare; known from a single collection on East Anacapa in October 1979 (large plant growing on mixed fill and gravel pile which had been brought to the island in early February 1979 [George Leone, personal communication, 1979]). EA, 26 Oct 1979, Junak \& Philbrick EA-27.

Ambrosia chamissonis (Less.) Greene silver beachbur Rare; known only from beach at Frenchy's Cove on West Anacapa.

WA, 9 May 1963, Blakley 5793; WA, 6 Apr 1968, Philbrick \& Osborne B68-138.

Artemisia californica Less. coastal sagebrush or california sagebrush Common; flats and slopes on all islets.

WA, 18 May 1961, Blakley 4383 (RSA, SBBG); MA, 9 May 1963, Piehl 63-200; EA, 26 Oct 1979, Junak d Philbrick EA-31.

Baccharis pilularis DC. subsp. consanguinea (DC.) C.B. Wolf coyote brush Occasional on flats and in canyons of West Anacapa; rare on flats of Middle and East Anacapa.

WA, 9 May 1963, Blakley 5757; MA, 12 May 1963, Piehl 63-283; EA, 5 Aug 1974, Philbrick B74-152.

Baccharis plummerae A. Gray subsp. plummerae Plummer's baccharis Rare; known from a single collection just northeast of Camel Peak on West Anacapa.

WA, 27 Oct 1979, Junak \& Philbrick WA-90 (JEPS, SBBG).

Baccharis salicifolia (Ruiz \& Pav.) Pers. subsp. salicifolia mule fat Rare; moist flats and slopes near Frenchy's Cove on West Anacapa.

WA, 9 May 1963, Blakley 5793 (CAS, RSA, SBBG).

Brickellia californica (Torr. \& A. Gray) A. Gray California brickell-bush or California boneset Rare; slopes on West Anacapa.

WA, 19 Mar 1967, Philbrick \& Ricker B67-27; WA, 2 October 1978, Junak \& Timbrook WA-7.

*Centaurea melitensis L. tocalote Scarce; disturbed flats and slopes along trail on West Anacapa and on disturbed flats in campground on East Anacapa. First collected on west flanks of Summit Peak on West Anacapa in June 1992.

WA, 3 Jun 1992, Junak WA-255 (RSA, SBBG); EA, 9 Jul 1998, Junak EA-169.

Corethrogyne filaginifolia (Hook. \& Arn.) Nutt. [Lessingia f. (Hook. \& Arn.) M.A. Lane] Californiaaster or cudweed-aster Common; flats and north-facing slopes on West and Middle Anacapa; scarce on north-facing slopes on East Anacapa.

WA, 11 May 1963, Blakley 5801; MA, 28 Oct 1979, Junak MA-38.

* Cotula australis (Spreng.) Hook.f. Australian cotula Scarce; disturbed flats on all islets. First collected near visitor's center building on East Anacapa in March 1979.

WA, 8 May 1980, Junak \& Philbrick WA-155; MA, 1 May 1986, Junak MA-189; EA, 30 Mar 1979, Junak EA-11.

* Cotula coronopifolia L. brass buttons Rare; flats on West and East Anacapa. First collected just east of the mouth of Box Canyon on West Anacapa in May 1980.

WA, 8 May 1980, Junak \& Philbrick WA-158; EA, 23 Apr 1982, Junak EA-64; EA, 11 May 1984, Pritchett 101.

Deinandra clementina (Brandegee) B.G. Baldwin [Hemizonia c. Brandegee] island tarplant Common; flats on Middle and East Anacapa. ENDEMIC to Santa Cruz, Anacapa, Santa Barbara, San Nicolas, Santa Catalina, and San Clemente islands.

MA, 28 May 1963, Blakley 5936 (CAS, SBBG); EA, 12 May 1984, Pritchett 109. 
Deinandra fasciculata (DC.) Greene [Hemizonia $f$. (DC.) Torr. \& A. Gray] common tarplant Scarce; flats on west end of West Anacapa.

WA, 3 Jun 1992, Junak WA-247 (RSA, SBBG).

*Delairea odorata Lam. [Senecio mikanioides Walp.] cape ivy Scarce; onshore slopes and canyons on West Anacapa. First collected in lower portion of Summit Canyon on that islet in November 2007.

WA, November 2007, Chaney \& Rodriguez s.n.

Encelia californica Nutt. bush sunflower Common on south-facing slopes of West and Middle Anacapa and along edges of terraces on Middle Anacapa; occasional on south-facing slopes and near center of terrace on East Anacapa.

WA, 27 Oct 1979, Junak \& Philbrick WA-84; MA, 7 Apr 68, Philbrick \& Osborne B68-166 (CAS, SBBG); EA, 26 Apr 1959, Blakley 2815 (JEPS, RSA, SBBG, SD).

*Erigeron bonariensis L. [Conyza b. (L.) Cronquist] flax-leaved fleabane Scarce; flats on West and East Anacapa. First collected in Cherry Canyon on West Anacapa in September 1964.

WA, 6 Sep 1964, Blakley 6518; EA, 5 Aug 1974, Philbrick B74-148.

Erigeron canadensis L. [Conyza c. (L.) Cronquist] horseweed Rare; flats on West and East Anacapa. WA, 1 Oct 1978, Junak et al. WA-1; EA, 23 Aug 1978, Junak \& Hochberg s.n. (SBBG 53423).

Erigeron foliosus var. foliosus Nutt. [E. $f$. var stenophyllus (Nutt.) A. Gray] leafy fleabane Rare; flats and slopes on West and Middle Anacapa.

WA, 10 May 1963, Piehl 63-238; MA, 9 May 1963 Blakley 5720 (CAS, SBBG).

Erigeron glaucus Ker Gawl. seaside daisy Occasional; north-facing slopes and terrace edges on all islets. The populations on Anacapa Island represent the southeastern-most occurrence of this taxon in the wild.

WA, 18 May 1961, Blakley 4333 (JEPS, RSA, SBBG); MA, 9 May 1963, Blakley 5740; EA, 24 Apr 1959, Blakley 2754 (CAS, JEPS, RSA, SBBG).

*Erigeron sumatrensis Retz. [Conyza floribunda Kunth, misapplied] tropical horseweed Rare; canyon bottom on West Anacapa and flats on East Anacapa. First collected on north side of West Anacapa in September 2012.

WA, 27 Sept 2012, Junak WA-461; EA, 29 Sept 2017 , Junak EA-175.

Eriophyllum confertiflorum (DC.) A. Gray var. confertiflorum golden-yarrow Common; flats and slopes on all islets.

WA, 18 May 1961, Blakley 4409 (RSA, SBBG); MA, 18 May 1961, Muller 1185; EA, 26 Apr 1959, Blakley 2797 (JEPS, RSA, SBBG).

Eriophyllum staechadifolium Lag. seaside woolly sunflower Occasional; north-facing slopes on all islets.
WA, 10 May 1963, Piehl 63-236; MA, 18 May 1961, Muller 1163 (RSA, SBBG); EA, 25 Apr 1959, Blakley 2779 .

Euthamia occidentalis Nutt. [Solidago o. (Nutt.) Torr. \& A. Gray] western goldenrod Rare; known from a single collection in Cherry Canyon on West Anacapa.

WA, 3 Oct 1978, Junak et al. WA-19.

Gamochaeta ustulata (Nutt.) Holub [Gnaphalium purpureum L., misapplied] purple cudweed Scarce; north-facing slopes in Cherry and Willow canyons on West Anacapa.

WA, 9 May 1963, Blakley 5774 (RSA, SBBG); WA, 3 Oct 1978, Junak \& Hochberg WA-25.

Grindelia stricta DC. var. platyphylla (Greene) M.A. Lane gumplant Abundant; flats and gentle slopes on all islets.

WA, 6 Sep 1964, Philbrick \& Flinck B64-319 (CAS, RSA, SBBG); MA, 5 Apr 1962, Blakley 5015; EA, 24 Apr 1959, Blakley 2743 (CAS, NY, RSA, SBBG, SD, US).

Hazardia detonsa (Greene) Greene [Haplopappus $d$. (Greene) Raven] northern island hazardia Common; north-facing slopes and flats on West Anacapa and extreme west end of Middle Anacapa. ENDEMIC to Santa Rosa, Santa Cruz, and Anacapa islands. Hybridizes with Hazardia squarrosa var. grindelioides.

WA, 3 Oct 1978, Junak et al. WA-15; MA, 23 Nov 1988, Junak MA-216 (RSA, SBBG).

Hazardia squarrosa (Hook. \& Arn.) Greene var. grindelioides (DC.) W.D. Clark [Haplopappus $s$. Hook. \& Arn. subsp. g. (DC.) D.D. Keck] sawtoothed goldenbush Scarce; slopes and flats on West Anacapa.

WA, 2 Oct 1978, Junak \& Timbrook WA-11.

*Helianthus annuus L. common sunflower Rare; known from a single collection on flats at west end of West Anacapa in September 1986.

WA, 23 Sep 1986, Drost 393.

*Hypochaeris glabra L. smooth cat's-ear Rare; flats and slopes on all islets. First collected on grassy slope just west of lighthouse on East Anacapa in April 1970.

WA, 6 May 1980, Junak \& Philbrick WA-118; MA, 28 Feb 1980, Junak \& Philbrick MA-44; EA, 22-23 Apr 1970, Benedict s.n. (SBBG 54303).

Isocoma menziesii (Hook. \& Arn.) G.L. Nesom var. sedoides (Greene) G.L. Nesom [Haplopappus venetus (Kunth) S.F. Blake var. $s$. (Greene) Munz] prostrate coastal goldenbush Rare; flats on East Anacapa.

EA, 26 Apr 1959, Blakley 2822

Isocoma menziesii (Hook. \& Arn.) G.L. Nesom var. vernonioides (Nutt.) G.L. Nesom coastal goldenbush [Haplopappus v. (Kunth) S.F. Blake var. v.] Occasional; flats and slopes on all islets. 
WA, 16 Nov 1969, Blakley 7025; MA, 7 Sep 1964, Blakley 6529; EA, 17 Jan 1985, Junak \& Fowler EA-83.

*Lactuca serriola L. prickly lettuce Rare; known from 2 occurrences: on a slope in the upper east fork of Box Canyon on West Anacapa and in open grassland on the ridge near the west end of Middle Anacapa. Both occurrences were very small populations (2 juvenile plants on West Anacapa and 12 adults and 1 juvenile plant on Middle Anacapa); all plants seen were removed. First collected on West Anacapa in May 1980.

WA, 5 May 1980, Junak \& Philbrick WA-151; MA, 16 Jul 2001, Junak \& Rosen MA-426.

Lasthenia gracilis (DC.) Greene common goldfields Occasional; flats and slopes on all islets.

WA, 4 Apr 1996, Junak WA-416 (RSA, SBBG); MA, 23 Apr 1982, Junak \& Philbrick MA-47; EA, 25 Apr 1959, Blakley 2774 (JEPS, NY, RSA, SBBG, SD, US).

Leptosyne gigantea Kellogg [Coreopsis g. (Kellogg) H.M. Hall] giant coreopsis Abundant; flats and slopes, especially north-facing, on all islets.

WA, 27 Mar 1965, Philbrick B65-503; EA, 24 Apr 1959, Blakley 2761 (CAS, RSA, SBBG, SD).

Logfia filaginoides (Hook. \& Arn.) Morefield [Filago californica Nutt.] california cotton-rose Scarce; open flats and slopes on West and Middle Anacapa.

WA, 3 Apr 1996, Junak WA-402; MA, 5 Apr 1995, Junak MA-302 (RSA, SBBG).

Malacothrix foliosa A. Gray subsp. crispifolia W.S. Davis Anacapa Island chicory Rare; flats and gentle slopes on extreme eastern end of West Anacapa and on East Anacapa. ENDEMIC to Anacapa Island.

WA, 31 Mar 1962, Blakley 4940; EA, 28 Apr 1993, Junak EA-119.

Malacothrix junakii W.S. Davis Junak's island chicory Rare; ledges and slopes on Middle Anacapa. ENDEMIC to Anacapa Island.

MA, 22 Apr 1986, Junak MA-105.

Malacothrix saxatilis (Nutt.) Torr. \& A. Gray var. implicata (Eastw.) H.M. Hall cliff malacothrix Occasional; flats and slopes on all islets. ENDEMIC to San Miguel, Santa Rosa, Santa Cruz, Anacapa, and San Nicolas islands.

WA, 18 May 1961, Muller 1173; MA, 27 Mar 1979, Junak \& Hochberg MA-28; EA, 22-23 Apr 1970, Benedict s.n. (SBBG 43139).

Malacothrix squalida Greene island malacothrix Rare; flats and gentle slopes on Middle Anacapa. ENDEMIC to Santa Cruz and Anacapa islands. MA, 30 Apr 1986, Junak MA-172.

*Matricaria discoidea DC. [Chamomilla suaveolens (Pursh) Rydb., M. matricarioides (Less.) Porter] pineapple weed Rare; known from a single collection near buildings on East Anacapa in March 1979.

EA, 27 Mar 1979, Junak \& Hochberg EA-6.

Perityle emoryi Torr. Emory's rock daisy Occasional; flats and slopes, especially south-facing, on all islets.

WA, 9 May 1963, Blakley 5749 (CAS, RSA, SBBG); MA, 9 May 1963, Blakley 5734; EA, 22-23 Apr 1970, Benedict s.n. (SBBG 43196).

Pseudognaphalium biolettii Anderb. [Gnaphalium bicolor Bioletti] bicolored everlasting Scarce; flats and slopes on all islets.

WA, 7 May 1980, Junak \& Philbrick WA-127; MA, 2 Jun 1978, Philbrick \& Hochberg B78-307; EA, 30 Mar 1979, Philbrick \& Hochberg B79-17b.

Pseudognaphalium californicum (DC.) Anderb. [Gnaphalium c. DC.] green everlasting Rare; known from two collections ("brushy slope" on unspecified islet and Cherry Canyon on West Anacapa).

A, 16 Mar 1929, Hoffmann s.n. (SBBG 80027); WA, 9 May 1963, Blakley 5776.

*Pseudognaphalium luteoalbum (L.) Hilliard \& B.L. Burtt [Gnaphalium l. L.] weedy cudweed Rare; known from a single collection in Cherry Canyon on West Anacapa in October 1978.

WA, 3 Oct 1978, Junak et al. WA-21.

Pseudognaphalium microcephalum (Nutt.) Anderb. [Gnaphalium canescens subsp. $m$. (Nutt.) Stebbins \& D.J. Keil] white everlasting Rare; flats and slopes on West and East Anacapa.

WA, 27 Oct 1979, Junak \& Philbrick WA-89; EA, 22-23 Apr 1970, Benedict s.n. (SBBG 43166).

Pseudognaphalium stramineum (Kunth) Anderb. [Gnaphalium chilense Spreng., Gnaphalium s. Kunth] cotton-batting Scarce; flats and slopes on all islets.

WA, 9 Apr 1998, Junak WA-443 (RSA, SBBG); MA, 1 May 1986, Junak MA-192; EA, 24 Apr 1959, Blakley 2750 (CAS, RSA, SBBG).

Rafinesquia californica Nutt. California chicory Rare; flats and slopes on West and Middle Anacapa.

WA, 3 Jun 1992, Junak WA-249 (RSA, SBBG); MA, 6 Apr 1995, Junak MA-317.

*Senecio vulgaris L. common groundsel Rare; flats on all islets. First collected near east end of East Anacapa, above Arch Rock in April 1959.

WA, 28 Mar 1979, Junak \& Hochberg WA-46; MA, 23 Apr 1982, Junak \& Philbrick MA-51; EA, 25 Apr 1959, Blakley 2769.

*Silybum marianum (L.) Gaertn. milk thistle Rare; disturbed flats on East Anacapa. First collected near the northern edge of the water catchment pad on East Anacapa in March 1995. All plants seen were removed at that time. The taxon was 
seen again near the campground in March 2015.

EA, 1 March 1995, Chaney SC495.

*Sonchus asper (L.) Hill prickly sow thistle Rare; flats on West and East Anacapa. First collected at edge of water catchment area on East Anacapa in April 1959.

WA, 9 May 1963, Blakley 5769 (RSA, SBBG); EA, 26 Apr 1959, Blakley 2795 (CAS, SBBG).

*Sonchus oleraceus L. common sow thistle Common; flats and slopes on all islets. First collected on island (islet and exact location not specified) in 1901.

A, 1901, Hemphill s.n. (UC 134386); WA, 18 May 1961, Blakley 4386 (CAS, RSA, SBBG, SD); MA, 31 Mar 1962, Blakley 4907 (RSA, SBBG); EA, 24 Apr 1959, Blakley 2746 (CAS, JEPS, SBBG).

Stebbinsoseris heterocarpa (Nutt) K.L. Chambers [Microseris $h$. (Nutt.) K.L. Chambers] Rare; known from 2 collections on terrace just southwest of Sheep Camp on Middle Anacapa.

MA, 22 Apr 1986, Junak MA-86; MA, 5 May 1998, Junak MA-387.

Uropappus lindleyi (DC.) Nutt. [Microseris lindleyi (DC.) A. Gray, M. linearifolia (DC.) Sch.Bip.] silver puffs Occasional; flats and slopes on West and Middle Anacapa.

WA, 1 Apr 1962, Blakley 4999 (RSA, SBBG); MA, 9 May 1963, Piehl 63-185 (CAS, SBBG).

\section{BERBERIDACEAE \\ (Barberry Family)}

Berberis pinnata Lagasca subsp. insularis Munz [Mahonia p. (Lagasca) Fedde subsp. $i$. (Munz) Roof] island barberry Rare; most recently known from a single colony in Summit Canyon on West Anacapa and now presumed to be extinct on the island, where it was last seen alive in August 1990. ENDEMIC to Santa Rosa (last collected there in 1930), Santa Cruz, and Anacapa islands.

WA, 26 Aug 1940, Dunkle 7668 (SBBG, SD); WA, 7 May 1980, Junak \& Philbrick WA-141 (CAS, RSA, SBBG); WA, 30 Aug 1990, Junak WA-240; WA, 15 Nov 1994, Junak \& Chaney WA-335 (branches of dead shrub).

\section{BORAGINACEAE (Borage Family)}

Amsinckia intermedia Fisch. \& C.A. Mey. common fiddleneck Scarce; flats and slopes on all islets.

WA, 28 Mar 1979, Philbrick B79-5; MA, 2 Apr 1998, Junak \& Kirkland MA-349 (RSA, SBBG); EA, 22 Apr 1970, Benedict s.n. (SBBG 43419).

Amsinckia menziesii (Lehm.) A. Nelson \& J.F. Macbr. small-flowered fiddleneck Rare; flats on Middle Anacapa.

MA, 1 Apr 1994, Junak MA-291.
Amsinckia spectabilis Fisch. \& C.A. Mey. var. spectabilis seaside fiddleneck Rare; flats and gentle slopes on all islets.

WA, 22 Apr 1970, Benedict s.n. (SBBG 43398); MA, 30 Apr 1986, Junak MA-170; EA, 26 Apr 1959, Blakley 2790 (CAS)

Cryptantha clevelandii Greene var. florosa I.M. Johnst. coastal cryptantha Occasional; flats on West and Middle Anacapa.

WA, 20 Mar 1997, Junak \& Kirkland WA-434; MA, 2 Apr 1998, Junak \& Kirkland MA-350.

Eucrypta chrysanthemifolia (Benth.) Greene var. chrysanthemifolia Occasional; flats and slopes, especially along northern edge of terraces, on West and Middle Anacapa.

WA, 18 Mar 1941, Moran \& Templeton 725 (RSA, SBBG); MA, 31 Mar 1962, Blakley 4915 (JEPS, SBBG).

Heliotropium curassavicum L. var oculatum (A. Heller) Tidestr. seaside heliotrope or alkali heliotrope Rare; flats and slopes on West and Middle Anacapa. Not seen recently.

WA, 18 May 1961, Muller 1174; MA, 1 Aug 1938, Elmore 222 (POM).

Pectocarya linearis (Ruiz \& Pav.) DC. subsp. ferocula (I.M. Johnst.) Thorne slender comb-seed Scarce; open flats and slopes along main ridge on West Anacapa.

WA, 3 Apr 1996, Junak WA-401.

Phacelia cicutaria Greene var. hispida (A. Gray) J.T. Howell caterpillar phacelia Scarce; flats and north-facing slopes on West and Middle Anacapa.

WA, 28 Mar 1965, Philbrick B65-521; MA, 29 Apr 1986, Junak MA-154.

Phacelia distans Benth. wild-heliotrope Occasional; flats and gentle slopes on all islets.

WA, 18 Mar 1941, Moran 721 (RSA, SBBG); MA, 2 Apr 1998, Junak \& Kirkland MA-353 (RSA, SBBG); EA, 24 Apr 1959, Blakley 2749 (OBI, SBBG).

Phacelia viscida (Lindl.) Torr. var. viscida sticky phacelia Rare; rocky slopes on West and Middle Anacapa.

WA, 11 May 1963, Blakley 5795 (RSA, SBBG); MA, 30 Apr 1986, Junak MA-169.

Plagiobothrys canescens Benth. var. catalinensis (A. Gray) Jeps. Santa Catalina popcorn-flower Rare; flats and ridgetops near Camel Peak on West Anacapa.

WA, 1 Apr 1962, Blakley 4977a.

Plagiobothrys collinus (Phil.) I.M. Johnst. var. gracilis (I.M. Johnst.) Higgins San Diego popcornflower Scarce; flats and ridgetops on West and Middle Anacapa.

WA, 3 Apr 1996, Junak WA-407; MA, 31 Mar 1962, Blakley 4927. 
BRASSICACEAE

(Mustard Family)

*Brassica nigra (L.) W.D.J. Koch black mustard Rare; known from a single collection at water catchment pad on East Anacapa in February 1980.

EA, 27 Feb 1980, Junak \& Philbrick EA-36.

*Cakile maritima Scop. sea rocket Rare; rocky beach at Frenchy's Cove on West Anacapa. First collected there in May 1963.

WA, 11 May 1963, Blakley 5794 (JEPS, RSA, SBBG, US).

Cardamine californica (Nutt.) Greene [Dentaria $c$. Nutt.] milk maids Rare; on north-facing slopes in and near Eucalyptus grove at Sheep Camp landing on Middle Anacapa.

MA, 31 Mar 1962, Blakley 4891 (CAS, JEPS, RSA, SBBG, US).

Caulanthus lasiophyllus (Hook. \& Arn.) Payson [Guillenia l. (Hook. \& Arn.) Greene] California mustard Occasional; flats and slopes on West and Middle Anacapa.

WA, 31 Mar 1962, Blakley 4941 (CAS, RSA, SBBG); MA, 9 May 1963, Blakley 5739 (RSA, SBBG).

Descurainia pinnata (Walter) Britton subsp. pinnata tansy mustard Rare; known from a single collection "east of the top of the highest peak" on West Anacapa in April 1962. Not seen recently.

WA, 1 Apr 1962, Blakley 4980.

Erysimum insulare Greene island wallflower Rare; slopes on West Anacapa and flats, especially those with shell middens, on East Anacapa. ENDEMIC to San Miguel, Santa Rosa, Santa Cruz, and Anacapa islands.

WA, 11 May 1963, Blakley 5800 (CAS, JEPS, RSA, SBBG); EA, 27 Feb 1980, Junak \& Philbrick EA-45.

Hornungia procumbens (L.) Hayek [Hutchinsia p. (L.) Desv.] Rare; slopes on north side of West and Middle Anacapa.

WA, 5 Apr 1962, Smith 6654; MA, 23 Apr 1982, Junak \& Philbrick MA-46.

*Lepidium didymum L. [Coronopus d. (L.) Sm.] lesser swine cress Rare; north-facing slopes east of Sheep Camp landing on Middle Anacapa, in seagull nesting area. Also known from East Anacapa on east side of Cathedral Cove and at top of stairs at Landing Cove. First collected on island in April 1991.

MA, 30 Apr 1991, Junak MA-237 (RSA, SBBG); EA, 15 Mar 2010, Chaney SC493.

Lepidium nitidum Nutt. shining peppergrass Occasional; flats on West and Middle Anacapa.

WA, 1 Apr 1962, Blakley 4961 (CAS, JEPS, RSA, SBBG, US); MA, 2 Apr 1998, Junak \& Kirkland MA-351.

Lepidium oblongum Small [L. o. var. insulare C.L. Hitchc.] lentejilla Occasional; flats and slopes on all islets.
WA, 11 May 1963, Blakley 5808; MA, 30 Apr 1991, Junak MA-234 (CAS, RSA, SBBG, SD); EA, 2 Mar 1993, Junak EA-98.

*Sisymbrium irio L. London rocket Rare; flats and slopes on West and Middle Anacapa. First collected near Frenchy's Cove on West Anacapa in May 1963.

WA, 11 May 1963, Piehl 63-277 (RSA, SBBG); MA, 26 Mar 1979, Junak MA-10.

* Sisymbrium orientale L. Occasional on Middle Anacapa, especially on disturbed flats along northern edge of terrace. Rare on East Anacapa, where it is known from a single collection. First collected near northeast end of water catchment pad on East Anacapa in February 1980.

MA, 29 Apr 1986, Junak MA-130 (CAS, RSA, SBBG); EA, 27 Feb 1980, Junak \& Philbrick EA-35a.

\section{Cactaceae \\ (Cactus Family)}

Cylindropuntia prolifera (Engelm.) F.M. Knuth [Opuntia p. Engelm.] coastal cholla Occasional; southern edges of terraces and south-facing slopes on all islets.

EA, 24 Apr 1959, Blakley 2762.

*Opuntia ficus-indica (L.) Mill. mission pricklypear Rare; slopes near Sheep Camp landing on Middle Anacapa. First collected on island "near abandoned ranch" (islet not specified, but probably Middle Anacapa) in June 1930.

A, 15 Jun 1930, Hoffmann 6528.

Opuntia littoralis (Engelm.) Cockerell coastal prickly-pear Occasional; flats and south-facing slopes on all islets. Hybridizes with $O$. oricola.

EA, 26 Apr 1959, Blakley 2823 (SBBG, SD).

Opuntia oricola Philbrick tall prickly-pear Occasional; flats and south-facing slopes on all islets.

MA, 1 Aug 1938, Elmore 245 (RSA).

\section{Caryophyllaceae (Pink Family)}

*Cerastium glomeratum Thuill. sticky mouse-ear chickweed Rare; north-facing slope above Sheep Camp landing on Middle Anacapa. First collected there in April 1991.

MA, 30 Apr 1991, Junak MA-238.

*Polycarpon tetraphyllum (L.) L. var. tetraphyllum four-leaved all-seed Rare; along trail at Sheep Camp landing on Middle Anacapa. First collected there in April 1986.

MA, 29 Apr 1986, Junak MA-118.

* Silene gallica L. windmill pink Occasional; open flats and slopes on all islets. First collected on West Anacapa (exact location not specified) in May 1940. 
WA, 16 May 1940, Bond 519; MA, 31 Mar 1962 Blakley 4899 (CAS, JEPS, NY, RSA, SBBG, SD, US); EA, 26 Apr 1959, Blakley 2798.

Silene laciniata Cav. subsp. laciniata [S.l. subsp. major C.L. Hitchc. \& Maguire] mexican pink Occasional; north-facing slopes on all islets.

WA, 18 May 1961, Blakley 4373 (CAS, JEPS, RSA, SBBG); MA, 18 May 1962, Muller 1177; EA, 26 Oct 1979, Junak \& Philbrick EA-30.

* Spergularia bocconi (Scheele) Graebn. boccone's sand-spurrey Scarce; along trails and on disturbed flats on Middle and East islets. First collected at campsite above Sheep Camp landing on Middle Anacapa in June 1992.

MA, 2 Jun 1992, Junak MA-245 (RSA, SBBG); EA, 6 Apr 1993, Junak EA-107.

Spergularia macrotheca (Cham. \& Schltdl.) Heynh. subsp. macrotheca sticky sand-spurrey Occasional; terrace edges and slopes, especially northfacing, on all islets.

WA, 10 Apr 1998, Junak WA-454; MA, 31 Mar 1962, Blakley 4879 (RSA, SBBG); EA, 26 Apr 1959, Blakley 2785 (CAS, JEPS, RSA, SBBG, SD, US).

Spergularia marina (L.) Besser saltmarsh sandspurrey Rare; disturbed flats on East islet.

EA, 13 Apr 1984, Drost 385.

* Stellaria media (L.) Vill. common chickweed Occasional; flats and north-facing slopes on all islets. First collected above Sheep Camp landing on Middle Anacapa in March 1979.

WA, 8 May 1980, Junak \& Philbrick WA-154 (RSA, SBBG); MA, 25 Mar 1979, Philbrick s.n. (SBBG 53326); EA, 4 Apr 1995, Junak EA-133.

\section{Chenopodiaceae \\ (Goosefoot Family)}

Aphanisma blitoides Moq. aphanisma Rare; southfacing slopes at edge of terrace on Middle Anacapa.

MA, 2 Jun 1992, Junak MA-249 (CAS, RSA, SBBG); MA, 19 Mar 1997, Junak MA-342.

Atriplex californica Moq. California saltbush Occasional; slopes and flats, especially at edges of terraces, on all islets.

WA, 31 Mar 1994, Junak WA-328 (RSA, SBBG); MA, 1 Apr 1994, Junak MA-270 (RSA, SBBG); EA, 18 Mar 1996, Junak EA-149 (RSA, SBBG).

Atriplex coulteri (Moq.) D. Dietr. Coulter's saltscale Rare; open sites at southern edge of terrace on Middle Anacapa.

MA, 30 Apr 1991, Junak MA-233 (CAS, RSA, SBBG).

Atriplex lentiformis (Torr.) S. Watson subsp. breweri (S. Watson) H.M. Hall \& Clem. Brewer's saltbush Scarce; flats and slopes on all islets.

WA, 23 Apr 1982, Junak WA-196; MA, 1 Nov 1988, Junak MA-200 (CAS, RSA, SBBG); EA, 26 Apr 1959, Blakley 2794 (CAS, JEPS, NY, RSA, SBBG, SD, US).
Atriplex leucophylla (Moq.) D. Dietr. seascale Rare; known from a single collection on "steep rocky slope” on Middle Anacapa. Not seen recently.

MA, 1 Aug 1938, Elmore 237 (RSA).

Atriplex pacifica A. Nelson south coast saltscale Scarce; flats and slopes on West and Middle Anacapa.

WA, 3 Apr 1996, Junak WA-403 (CAS, RSA, SBBG); MA, 30 Apr 1986, Junak MA-166 (CAS, SBBG).

*Atriplex semibaccata R. Br. Australian saltbush Common; disturbed flats and slopes on all islets. First collected on island in June 1930 (exact location and islet not specified).

A, 16 Jun 1930, Hoffmann s.n. (SBBG 77630); WA, 18 May 1961, Blakley 4357 (CAS, JEPS, RSA, SBBG, US); MA, 28 Oct 1979, Junak et al. MA-40 (RSA, SBBG); EA, 25 Apr 1959, Blakley 2772 (CAS, JEPS, RSA, SBBG, SD).

Atriplex watsonii Abrams matscale Rare; northwestern portion of terrace on East Anacapa.

EA, 30 Mar 1979, Philbrick \& Hochberg B79-18.

*Beta vulgaris L. subsp. maritima (L.) Arcang. Sea beet Rare; known from a single collection at Frenchy's Cove on West Anacapa in April 1968. WA, 6 Apr 1968, Philbrick \& Osborne B68-131.

Chenopodium berlandieri Moq. var. sinuatum (Murr) Wahl pitted goosefoot Rare; disturbed flats and gentle slopes on Middle and East Anacapa.

MA, 1 Nov 1988, Junak MA-198 (RSA, SBBG); EA, 25 Apr 1959, Blakley 2768 (JEPS, RSA, SBBG, SD, US).

Chenopodium californicum (S. Watson) S. Watson soaproot Occasional; flats and slopes on all islets. WA, 4 Apr 1996, Junak WA-412 (RSA, SBBG); MA, 30 Apr 1986, Junak MA-173; EA, 18 Mar 1996, Junak EA-148 (RSA, SBBG).

*Chenopodium murale L. nettle-leaf goosefoot Occasional; disturbed flats and slopes on all islets. First collected "near harbor for landing sheep” on Middle Anacapa in September 1930.

WA, 2 Oct 1978, Junak \& Timbrook WA-10; MA, 22 Sep 1930, Hoffmann s.n. (SBBG 77579); EA, 24 Apr 1959, Blakley 2751 (RSA, SBBG, US).

Salicornia pacifica Standl. [S. virginica L., misapplied] pickleweed Rare; known from a single collection on "sea cliff" on Middle Anacapa in August 1940.

MA, 21 Aug 1940, Dunkle 7652 (RSA, SBBG).

* Salsola tragus L. russian thistle or tumbleweed Common; flats and slopes on Middle Anacapa. First collected on western portion of Middle Anacapa terrace in July 2001, when less than 50 plants were seen. Most of the plants seen in 2001 were on a slope heavily disturbed by nesting sea gulls. This taxon has spread rapidly since then, especially during dry years.

MA, 17 Jul 2001, Junak \& Rosen MA-429. 
Suaeda taxifolia (Standl.) Standl. [S. californica S. Watson var. pubescens Jeps.; S. c. var. taxifolia (Standl.) Munz] woolly seablite Occasional; flats, especially along southern edges of terraces, and on slopes around perimeter of all islets.

WA, 27 Mar 1965, Philbrick B65-505; MA, 1 Nov 1988, Junak MA-201 (RSA, SBBG, SD); EA, 21 May 1982, Junak EA-79.

\section{Convolvulaceae \\ (Morning Glory Family)}

Calystegia macrostegia (Greene) Brummitt subsp. macrostegia island morning-glory Common; flats and slopes on all islets. ENDEMIC to San Miguel, Santa Rosa, Santa Cruz, Anacapa, Santa Catalina, San Martin, and Guadalupe islands.

WA, 18 May 1961, Muller 1166; MA, 29 Apr 1986, Junak MA-135 (CAS, RSA, SBBG, SD); EA, 24 Apr 1959, Blakley 2763 (CAS, RSA, SBBG, US).

Cressa truxillensis Kunth alkali weed Rare; flats on Middle Anacapa.

MA, 9 May 1963, Piehl 63-199 (RSA, SBBG).

Cuscuta salina Engelm. salt marsh dodder Rare; flats at western end of terrace on East Anacapa. Host plants for this parasitic plant include Dudleya caespitosa, Frankenia salina, and Suaeda taxifolia on Anacapa.

EA, 26 Apr 1959, Blakley 2811 (CAS, JEPS, RSA, SBBG); EA, 17 Sep 1978, Hochberg s.n. (SBBG 54282, SBBG 54283).

\section{Crassulaceae \\ (Stonecrop Family)}

Crassula connata (Ruiz \& Pav.) A. Berger [Tillaea erecta Hook. \& Arn.] pygmy weed Rare; open sites on flats and slopes on all islets.

WA, 10 Apr 1998, Junak WA-455 (RSA, SBBG); MA, 30 Apr 1991, Junak MA-222 (RSA, SBBG); EA, 26 Apr 1959, Blakley 2805.

Dudleya caespitosa (Haw.) Britton \& Rose sandlettuce Abundant; flats and slopes on all islets.

WA, 18 May 1961, Blakley 4411 (CAS, JEPS, RSA, SBBG); MA, 27 Mar 1965, Philbrick B65-469a; EA, 26 Apr 1959, Blakley 2808 (CAS, JEPS, RSA, SBBG, SD, US).

\section{Cucurbitaceae (Gourd Family)}

Marah macrocarpa (Greene) Greene [M. m. var. major (Dunn) Stocking] chilicothe or wild cucumber Occasional; flats and north-facing slopes on all islets.

WA, 13 Jan 1994, Junak WA-263 (RSA, SBBG, SD, UC, UCD); MA, 21 Mar 1996, Junak MA-329 (RSA, SBBG, SD); EA, 24 Apr 1959, Blakley 2760 (JEPS, RSA, SBBG).

\author{
ERICACEAE \\ (Heather Family)
}

Comarostaphylis diversifolia (Parry) Greene subsp. planifolia (Jeps.) G.D. Wallace summer holly Rare; known from a single individual found on a northeast-facing slope in upper east fork of Oak Canyon on West Anacapa.

WA, 9 May 1985, Junak et al. WA-218.

\section{EuPHORBIACEAE \\ (Spurge Family)}

*Chamaesyce maculata (L.) Small spotted spurge Rare; known from a single collection at landing on East Anacapa. First collected there in March 1996.

EA, 18 Mar 1996, Junak EA-145.

Euphorbia spathulata Lam. Rare; known from a single population on gentle west-facing slope on terrace in the southwestern portion of West Anacapa.

WA, 10 Apr 1998, Junak WA-458 (RSA, SBBG, UCR).

$$
\begin{aligned}
& \text { FABACEAE } \\
& \text { (Pea Family) }
\end{aligned}
$$

*Acacia longifolia (Andrews) Willd. golden wattle Rare; known from a single individual on northfacing slope below lighthouse on East Anacapa. First collected there in November 1988.

EA, 23 Nov 1988, Junak EA-93.

Acmispon dendroideus (Greene) Brouillet var. dendroideus [Lotus d. (Greene) Greene var. d., L. scoparius (Nutt.) Ottley var. $d$. (Greene) Ottley] island deerweed or island broom Common; flats and slopes on all islets. ENDEMIC to Santa Rosa, Santa Cruz, Anacapa, and Santa Catalina islands. Some plants on Anacapa approach var. veatchii of San Miguel Island.

WA, 13 Jan 1994, Junak WA-264 (CAS, RSA, SBBG, SD, UCD); MA, 22 Oct 1991, Junak MA-242 (CAS, RSA, SBBG, SD); EA, 21 May 1982, Junak EA-78.

Acmispon maritimus (Nutt.) D.D. Sokoloff var. maritimus [Lotus salsuginosus Greene var. $s$.] coastal lotus Scarce; open ridgetops and slopes on West and Middle Anacapa.

WA, 29 Mar 1995, Junak WA-353; MA, 4 May 1998, Junak MA-369 (RSA, SBBG).

Acmispon strigosus (Nutt.) Brouillet [Lotus s. (Nutt.) Greene] bishop's lotus Scarce; flats and slopes on Middle Anacapa.

MA, 5 May 1998, Junak MA-385 (RSA, SBBG, SD).

Acmispon wrangelianus (Fisch. \& C.A. Mey.) D.D. Sokoloff [Lotus w. Fisch. \& C.A. Mey., L. subpinnatus Lag., misapplied] Rare; known from a single collection on West Anacapa (exact location not specified) in May 1940.

WA, 16 May 1940, Bond 512. 
Astragalus didymocarpus Hook. \& Arn. var. didymocarpus [A. catalinensis Nutt.] common dwarf locoweed or two-seeded milkvetch Rare; flats and slopes on West Anacapa.

WA, 3 Apr 1996, Junak WA-406.

Astragalus miguelensis Greene San Miguel Island locoweed Occasional; flats and slopes on West and Middle Anacapa. ENDEMIC to San Miguel, Santa Rosa, Santa Cruz, Anacapa, and San Clemente islands.

WA, 6 May 1980, Junak \& Philbrick WA-110; MA 15 Jun 1982, Junak \& Philbrick MA-75.

Astragalus trichopodus (Nutt.) A. Gray var. lonchus (M.E. Jones) Barneby [A. t. subsp. leucopsis (Torr. \& A. Gray) Thorne] southern California locoweed Occasional; flats and slopes on all islets.

WA, 30 Mar 1994, Junak WA-308; MA, 30 Apr 1986, Junak MA-168; EA, 30 Mar 1979, Philbrick \& Hochberg B79-11.

Lathyrus vestitus Nutt. var. vestitus wild sweet pea Occasional; flats and slopes on West Anacapa.

WA, 9 May 1963, Blakley 5762 (CAS, RSA, SBBG, SD).

Lupinus albifrons Benth. var. douglasii (J. Agardh) C.P. Sm. silver bush lupine Occasional; flats and slopes on West Anacapa.

WA, 21 Mar 1941, Moran 738 (CAS, DS, RSA, SD, UCR).

Lupinus bicolor Lindl. dove lupine Occasional; flats and slopes on West and Middle Anacapa.

WA, 18 Mar 1967, Philbrick \& Ricker B67-8; MA, 5 Apr 1962, Smith 6667.

Lupinus hirsutissimus Benth. stinging lupine Rare; south-facing slopes, especially those with loose talus, on south side of West Anacapa, just west of Cat Rock.

WA, 29 Mar 1995, Junak WA-360.

Lupinus succulentus K. Koch arroyo lupine or succulent lupine Occasional; grassy flats and moist slopes on West and Middle Anacapa.

WA, 29 Mar 1994, Junak WA-273 (RSA, SBBG); MA, 5 Apr 1995, Junak MA-306 (RSA, SBBG).

Lupinus truncatus Nutt. truncate lupine Scarce; flats and rocky slopes on all islets.

WA, 28 Mar 1979, Junak \& Hochberg WA-65; MA, 22 Apr 1986, Junak MA-81; EA, 4 Apr 1995, Junak EA-141 (RSA, SBBG).

*Medicago polymorpha L. bur-clover Occasional; flats and slopes on all islets. First collected on northeastern side of West Anacapa in May 1961.

WA, 18 May 1961, Blakley 4372 (RSA, SBBG); MA, 31 Mar 1962, Blakley 4933; EA, 26 Oct 1979, Junak et al. EA-34.

*Medicago sativa L. alfalfa Rare; flats on Middle Anacapa. Known from a single collection from middle part of the islet in August 1940.

MA, 20 Aug 1940, Dunkle 7645 (POM, SBBG).
*Melilotus albus Medik. white sweet-clover Rare; known from a single collection at water catchment pad on East Anacapa in October 1979 (growing on mixed fill and gravel pile which had been brought to the island in early February 1979 [George Leone, personal communication, 1979]).

EA, 26 Oct 1979, Junak \& Philbrick EA-28.

*Melilotus indicus (L.) All. sour-clover Occasional; flats and slopes on all islets. First collected on island (exact location and islet not specified) in June 1930.

A, 15 Jun 1930, Hoffmann s.n. (SBBG 78336); WA, 11 May 1963, Piehl 63-281; MA, 17 May 1940, Bond 511; EA, 23 Apr 1982, Junak EA-65.

Trifolium depauperatum Desv. var. truncatum (Greene) Isely [T. amplectens Torr. \& A. Gray var. $t$. (Greene) Jepson] dwarf bladder clover Rare; moist flats and slopes on West and Middle Anacapa.

WA, 1 Apr 1962, Blakley 4970 (OBI, SBBG); MA, 26 Mar 1979, Junak \& Hochberg MA-5a.

Trifolium fucatum Lindl. var. gambelii (Nutt.) Jeps. bull clover Scarce; flats on East Anacapa.

EA, 6 Apr 1993, Junak EA-99 (RSA, SBBG, SD).

Trifolium gracilentum Torr. \& A. Gray pinpoint clover Scarce; flats and slopes on all islets.

WA, 8 Apr 1998, Junak WA-442 (RSA, SBBG); MA, 2 Apr 1998, Junak MA-345; EA, 28 Apr 1993, Junak EA-111.

Trifolium microcephalum (Pursh) Poir. small-headed clover Rare; flats and slopes on and near ridgetop of West Anacapa.

WA, 9 Apr 1998, Junak WA-448 (RSA, SBBG).

Trifolium palmeri S. Watson [T. gracilentum var. $p$. (S. Watson) McDermott] southern island clover Rare; slopes in southwestern portion of West Anacapa. ENDEMIC to Anacapa, Santa Barbara, San Nicolas, Santa Catalina, San Clemente, and Guadalupe islands.

WA, 2 Apr 1996, Junak WA-395 (RSA, SBBG).

Trifolium willdenovii Spreng. [T. tridentatum Lindl.] tomcat clover Occasional; flats and slopes on all islets.

WA, 9 May 1963, Blakley 5782 (RSA, SBBG); MA, 22 Apr 1986, Junak MA-79; EA, 26 Apr 1959, Blakley 2810.

*Vicia benghalensis L. purple vetch Rare; known from a single collection in Oak Canyon on West Anacapa in April 1987.

WA, 7 Apr 1987, Danielsen \& Halverson s.n. (SBBG 129245).

Vicia hassei S.Watson [V. exigua Nutt. var. $h$. (S. Watson) Jeps.] slender vetch Rare; slopes on north side of Middle Anacapa.

MA, 30 Apr 1986, Junak MA-159. 
Vicia ludoviciana Torr. \& A. Gray var. ludoviciana [V. exigua Nutt.] deer-pea vetch Rare; flats and slopes in southwestern portion of West Anacapa. WA, 20 Mar 1997, Junak WA-437 (RSA, SBBG).

*Vicia sativa L. subsp. sativa spring vetch Rare; known from a single collection near Sheep Camp landing on Middle Anacapa in April 1986. MA, 22 Apr 1986, Junak MA-87.

\section{FAGACEAE \\ (Oak Family)}

Quercus chrysolepis Liebm. canyon live oak Rare; lower portion of Oak Canyon on West Anacapa.

WA, 1 Oct 1978, Junak \& Hochberg WA-4 (CAS, SBBG).

Quercus tomentella Engelm. island oak Scarce; small localized groves in Oak Canyon on West Anacapa. ENDEMIC to Santa Rosa, Santa Cruz, Anacapa, Santa Catalina, San Clemente, and Guadalupe islands.

WA, 3 Jun 1951, Muller 8931.

\section{Frankeniaceae}

(Frankenia Family)

Frankenia salina (Molina) I.M. Johnst. [F. grandifolia Cham. \& Schltdl.] alkali heath Common; flats and coastal slopes on all islets.

WA, 11 May 1963, Blakley 5805; MA, 31 Mar 1962, Blakley 4878; EA, 28 Apr 1993, Junak EA-118 (RSA, SBBG).

\section{Geraniaceae \\ (Geranium Family)}

*Erodium cicutarium (L.) L'Her. redstem filaree Scarce; open and eroded areas on all islets. First collected on island (exact locality and islet not specified) in March 1929.

A, 16 Mar 1929, Hoffmann s.n. (SBBG 78444); WA, 10 May 1963, Piehl 63-249b; MA, 22 Apr 1986, Junak MA-93; EA, 26 Apr 1959, Blakley 2820.

*Erodium moschatum (L.) L'Her. whitestem filaree Occasional; flats and slopes on all islets. First collected above East Fish Camp on Middle Anacapa in March 1962.

WA, 1 Apr 1962, Blakley 4978; MA, 31 Mar 1962, Blakley 4932; EA, 27 Feb 1980, Junak \& Philbrick EA-42.

\section{Grossulariaceae \\ (Currant or Gooseberry Family)}

Ribes malvaceum Sm. var. malvaceum chaparral currant Rare; canyons on north side of West Anacapa.

WA, 18 May 1961, Blakley 4391 (CAS, RSA, SBBG).

\section{LAMIACEAE \\ (Mint Family)}

Salvia mellifera Greene black sage Common; ridgetops and slopes, especially on south side, of West Anacapa.

WA, 1 Apr 1962, Blakley 4982 (CAS, RSA, SBBG).

Stachys bullata Benth. wood-mint Occasional; canyons on north side of West Anacapa.

WA, 8 May 1980, Junak \& Philbrick WA-143 (RSA, SBBG).

\section{MaLVaCEAE \\ (Mallow Family)}

*Malva arborea (L.) Webb \& Berthel. [Lavatera a. L.] tree mallow Common; disturbed flats and slopes on Middle Anacapa. First collected near water tank building on East Anacapa in April 1999 , but not seen recently on that islet.

MA, 3 May 2000, Junak MA-414; MA, 15 Dec 2009, Junak \& Chaney MA-432; EA, 15 Apr 1999, Williams \& Chaney SC510

Malva assurgentiflora (Kellogg) M.F. Ray [L. $a$. Kellogg subsp. a.] malva rosa or northern island tree mallow Rare; slopes on northern side of West and Middle Anacapa. Populations on East Anacapa were planted there by the National Park Service. This Northern Island form is ENDEMIC to San Miguel and Anacapa islands; populations on Santa Rosa and other California islands have apparently been planted.

WA, 3 Oct 1978, Timbrook \& Philbrick 652; MA, 22 Sep 1930, Hoffmann s.n. (SBBG 79965).

* Malva parviflora L. cheeseweed Common; disturbed areas on all islets. First collected on north side of Middle Anacapa in March 1962 and at Frenchy's Cove on West Anacapa in May 1963.

WA, 11 May 1963, Piehl 63-278; MA, 31 Mar 1962 , Blakley 4934 (SBBG, UCR); EA, 27 Mar 1979, Junak \& Hochberg EA-5.

*Malva pseudolavatera Webb \& Berthel. [Lavatera cretica L.] cretan mallow Common; disturbed flats and slopes on Middle and East Anacapa. First collected near Sheep Camp landing on Middle Anacapa in March 1979; it has spread aggressively on Middle Anacapa since then. First collected along edges of water catchment pad on East Anacapa in April 1993.

MA, 27 Mar 1979, Junak et al. MA-12 (RSA, SBBG); EA, 6 Apr 1993, Junak EA-108 (SBBG, SD).

\section{Montiaceae}

(Miner's Lettuce Family)

Calandrinia menziesii (Hook.) Torr. \& A. Gray Red maids Scarce; flats and slopes on all islets, but extremely localized (especially on East Anacapa). 
WA, 4 Apr 1996, Junak WA-417 (RSA, SBBG); MA, 30 Apr 1991, Junak MA-228 (CAS, RSA, SBBG, SD); EA, 4 Apr 1985, Junak EA-134.

Cistanthe maritima (Nutt.) Hershk. [Calandrinia $m$. Nutt.] seaside cistanthe or sea kisses Rare; open ridgetops, terrace edges, and slopes on all islets. Abundance can be extremely variable; rare in most years.

WA, 29 Mar 1995, Junak WA-359; MA, 15 Jun 1982 , Junak \& Philbrick MA-74; EA, 28 May 2014, Chaney \& Green SC500.

Claytonia parviflora Hook. subsp. parviflora miner's lettuce Rare; known from a single collection "north of and below summit" on West Anacapa.

WA, 10 May 1963, Piehl 63-257 (in part; CAS 982487).

Claytonia perfoliata Willd. subsp. mexicana (Rydb.) John M. Miller \& K.L. Chambers miner's lettuce Occasional; moist flats and north-facing slopes on West and Middle Anacapa.

WA, 10 Apr 1998, Junak WA-457 (RSA, SBBG); MA, 4 May 1998, Junak MA-379 (RSA, SBBG).

Claytonia perfoliata Willd. subsp. perfoliata miner's lettuce Rare; north-facing slope near Sheep Camp landing on Middle Anacapa.

MA, 30 Mar 1995, Junak MA-296 (SBBG, UCR).

\section{Myrsinaceae \\ (Myrsine Family)}

*Lysimachia arvensis (L.) U. Manns \& Anderb. [Anagallis a. L.] scarlet pimpernel Rare; disturbed flats and slopes near Sheep Camp landing on Middle Anacapa and near buildings on East Anacapa. First collected on Middle Anacapa in June 1978.

MA, 1 Jun 1978, Philbrick \& Hochberg B78-291; EA, 5 Apr 1995, Junak EA-144.

\section{Mrrtaceae \\ (Myrtle Family)}

*Eucalyptus globulus Labill. blue gum Rare; northfacing slope above Sheep Camp landing on Middle Anacapa, and previously growing by water tank building on East Anacapa (trees were planted there as early as 1936 [Lighthouse Keeper logs at National Archives] and were dead by 1980). Trees on Middle Anacapa are visible in photographs taken in the late 1880 s (i.e., photographs from the Lorenzo Yates expedition).

MA, 7 Sep 1964, Philbrick \& Flinck B64-326; EA, 26 Apr 1959, Blakley 2793.

\section{NyCTAGINACEAE \\ (Four O’clock Family)}

Abronia maritima S. Watson sticky sand-verbena Rare; beach (presumably at Frenchy's Cove on West Anacapa) and rocky slopes on Middle Anacapa.
A, 19 May 1928, Howell 3796 (CAS); A, 22 Sep 1930, Hoffmann s.n. (SBBG 77661); MA, 1 Aug 1938, Elmore 243 (RSA).

Mirabilis laevis (Benth.) Curran var. crassifolia (Choisy) Spellenb. [M. californica A. Gray] wishbone bush Occasional; flats and slopes on all islets.

WA, 29 Mar 1994, Junak WA-341 (CAS, RSA, SBBG); MA, 31 Mar 1994, Junak MA-257 (RSA, SBBG, SD); EA, 26 Apr 1959, Blakley 2802 (CAS, JEPS, RSA, SBBG, SD).

\section{Oleaceae}

(Olive Family)

*Olea europaea L. olive Rare; known from a single collection of a juvenile plant in the upper portion of Box Canyon on West Anacapa in October 1978.

WA, 3 Oct 1978, F.G. Hochberg s.n. (SBBG 53450).

$$
\begin{gathered}
\text { ONAGRACEAE } \\
\text { (Evening Primrose Family) }
\end{gathered}
$$

Camissoniopsis micrantha (Spreng.) W.L. Wagner \& Hoch [Camissonia m. (Spreng.) P.H. Raven] small evening primrose Rare; known from a single collection from northern edge of terrace on Middle Anacapa.

MA, 30 Apr 1991, Junak MA-227 (RSA, SBBG).

Clarkia epilobioides (Torr. \& A. Gray) A. Nelson \& J.F. Macbr. Rare; known from a single collection in Cherry Canyon on West Anacapa.

WA, 9 May 1963, Blakley 5755 (RSA, SBBG, SD).

Epilobium canum (Greene) P.H. Raven subsp. canum [Zauschneria c. Greene] California-fuchsia Occasional; canyons and slopes on north side of West and Middle Anacapa.

WA, 5 Sep 1964, Philbrick \& Flinck B64-315 (JEPS, SBBG); MA, 1 Nov 1988, Junak MA-195 (RSA, SBBG).

\section{OROBANCHACEAE}

(Broomrape Family)

Castilleja affinis Hook. \& Arn. subsp. affinis red paintbrush Common; flats and north-facing slopes on all islets.

WA, 21 Mar 1941, Moran 742 (RSA, SBBG); MA, 5 Apr 1962, Smith 6660; EA, 24 Apr 1959, Blakley 2764 (RSA, SBBG).

Castilleja hololeuca Greene [C. lanata A. Gray subsp. h. (Greene) T.I. Chuang \& Heckard] island paintbrush Occasional; flats and slopes, especially south-facing, on West and Middle Anacapa. ENDEMIC to San Miguel, Santa Rosa, Santa Cruz, and Anacapa islands.

WA, 5 Apr 1962, Smith 6649; MA, 31 Mar 1994, Junak MA-258 (RSA, SBBG).

Orobanche fasciculata Nutt. clustered broomrape Rare; known from a single collection in Summit Canyon on West Anacapa.

WA, 7 May 1980, Junak \& Philbrick WA-142. 


\section{OXALIDACEAE \\ (Oxalis Family)}

* Oxalis pes-caprae L. Bermuda buttercup Rare; flats near campground on East Anacapa. First collected there in March 2010.

EA, March 2010, Chaney s.n.

\section{Papaveraceae \\ (Poppy Family)}

Eschscholzia ramosa (Greene) Greene island poppy Rare; flats just east of campground on East Anacapa. This taxon had not been seen on the island until Malephora crocea was removed from the area. ENDEMIC to San Miguel, Santa Rosa, Santa Cruz, Anacapa, Santa Barbara, San Nicolas, Santa Catalina, San Clemente, Los Coronados, Todos Santos, San Martin, Guadalupe, San Benito, Cedros, and Natividad islands.

EA, 3 May 2014, Chaney \& Green SC503.

Papaver heterophyllum (Benth.) Greene [Stylomecon h. (Benth.) G. Taylor] wind poppy Rare; north-facing slopes near Frenchy's Cove on West Anacapa.

WA, 5 Apr 1962, Smith 6652.

Platystemon californicus Benth. cream cups Rare; slopes on West Anacapa.

WA, 28 Mar 1965, Philbrick B65-515.

\section{Phrymaceae \\ (Lopseed Family)}

Mimulus aurantiacus Curtis var. parviflorus (Greene) D.M. Thomps. [Diplacus p. Greene, M. flemingii Munz] island monkey flower Occasional; northfacing slopes and canyons on West Anacapa. ENDEMIC to San Miguel, Santa Rosa, Santa Cruz, Anacapa, and San Clemente islands. Some plants on Anacapa with yellow-orange corollas appear to be hybrids with Mimulus aurantiacus var. pubescens, but that taxon has not been seen on the island.

WA, 28 Mar 1979, Junak \& Hochberg WA-48.

\section{Plantaginaceae \\ (Plantain Family)}

Antirrhinum nuttallianum A.DC. subsp. subsessile (A. Gray) D.M. Thomps. Nuttall's snapdragon Occasional; terrace edges and slopes on all islets.

WA, 8 May 1980, Junak \& Philbrick WA-156; MA, 1 Jun 1978, Philbrick \& Hochberg B78-293; EA, 22 Apr 1970, Benedict s.n. (SBBG 43155).

Keckiella cordifolia (Benth.) Straw climbing penstemon Scarce; Oak and Summit canyons on West Anacapa.

WA, 7 May 1980, Junak \& Philbrick WA-140 (CAS, SBBG).
Nuttallanthus texanus (Scheele) D.A. Sutton [Linaria canadensis (L.) Chaz., in part, misapplied] blue toadflax Scarce; ridgetops and flats on all islets.

WA, 9 Apr 1998, Junak WA-446; MA, 31 Mar 1962, Blakley 4896 (JEPS, RSA, SBBG, SD); EA, 4 Apr 1995, Junak EA-136.

*Plantago coronopus L. cut-leaved plantain Rare; known from a single collection on disturbed flats at campground on East Anacapa in July 1998; all plants seen were removed.

EA, 9 Jul 1998, Junak EA-164.

Plantago erecta E. Morris California plantain Occasional; flats and slopes, especially open rocky sites, on West Anacapa.

WA, 31 Mar 1995, Junak WA-394 (RSA, SBBG).

Plantago ovata Forssk. var. insularis (Eastw.) S.C. Meyers \& A. Liston [P. insularis Eastw.] Occasional; open flats and slopes on West Anacapa. WA, 3 Jun 1992, Junak WA-256.

\section{Plumbaginaceae (Plumbago Family)}

*Limonium perezii (Stapf) F.T. Hubb. sea-lavender or statice Scarce; flats and slopes on Middle Anacapa. First collected near south edge of terrace just west of high point on islet in April 1995.

MA, 5 Apr 1995, Junak MA-299; MA, 4 May 1998, Junak MA-377.

\section{Polemoniaceae (Phlox Family)}

Gilia clivorum (Jeps.) V.E. Grant purplespot gilia Scarce; flats and slopes on West and Middle Anacapa.

WA, 28 Mar 1965, Philbrick B65-547; MA, 5 Apr 1962 , Blakley 5013 (JEPS, RSA, SBBG).

Gilia nevinii A. Gray island gilia Occasional; flats and slopes on West and Middle Anacapa. ENDEMIC to Santa Rosa, Santa Cruz, Anacapa, Santa Barbara, San Nicolas, Santa Catalina, San Clemente, and Guadalupe islands.

WA, 28 Mar 1965, Philbrick B65-525; MA, 30 Apr 1986, Junak MA-183 (CAS, SBBG).

\section{Polygonaceae \\ (Buckwheat Family)}

Eriogonum arborescens Greene Santa Cruz Island buckwheat Common; flats and slopes on all islets. ENDEMIC to Santa Rosa, Santa Cruz, and Anacapa islands.

WA, 18 May 1961, Blakley 4343 (CAS, RSA, SBBG, SD); MA, 22 Oct 1991, Junak MA-244 (RSA, SBBG); EA, 26 Apr 1959, Blakley 2800 (CAS, JEPS, RSA, SBBG, SD, US). 
Eriogonum grande Greene var. grande island buckwheat Common; flats and slopes on all islets. ENDEMIC to Santa Cruz, Anacapa, Santa Catalina, and San Clemente islands.

WA, 18 May 1961, Blakley 4404; MA, 23 Oct 1990, Junak \& Halvorson MA-219 (RSA, SBBG); EA, 24 Apr 1959, Blakley 2756 (CAS, JEPS, SBBG).

*Polygonum aviculare L. subsp. depressum (Meisn.) Arcang. [P. arenastrum Boreau] common knotweed Occasional; along trails and in campground on East Anacapa. First collected near Visitor Center building in August 1988.

EA, 19 Aug 1988, Junak EA-92 (RSA, SBBG).

Pterostegia drymarioides Fisch. \& C.A. Mey. fairy mist or woodland threadstem Occasional; flats and north-facing slopes on all islets.

WA, 10 May 1963, Piehl 63-256; MA, 27 Mar 1965, Philbrick B65-493 (JEPS, SBBG).

*Rumex crispus L. curly dock Rare; flats and gullies on Middle Anacapa and disturbed flats on East Anacapa. First collected on island (exact location and islet not specified) in June 1930 .

A, 15 Jun 1930, Hoffmann s.n. (SBBG 77474); MA, 4 May 1998, Junak MA-378 (RSA, SBBG); EA, 9 Jul 1998, Junak EA-167.

\section{Primulaceae}

(Primrose Family)

Primula clevelandii var. insularis (H.J. Thomps.) Mast \& Reveal [Dodecatheon c. Greene subsp. insulare H.J. Thomps.] shooting star Scarce; flats and north-facing slopes on all islets.

WA, 28 Mar 1965, Philbrick B65-530; MA, 16 Mar 1941, Moran 713 (CAS, RSA, SBBG, SD, UCR); EA, 28 Apr 1993, Junak EA-112.

\section{RANUNCULACEAE \\ (Buttercup Family)}

Delphinium parryi A. Gray subsp. maritimum (Davidson) M.J. Warnock maritime larkspur Scarce; flats and north-facing slopes on all islets. WA, 8 May 1980, Junak \& Philbrick WA-174; MA, 16 Mar 1941, Moran 714 (DS, RSA, SBBG, SD, UCR); EA, 26 Apr 1959, Blakley 2803.

\section{RESEDACEAE \\ (Mignonette Family)}

Oligomeris linifolia (Hornem.) J.F. Macbr. oligomeris Scarce; ridgetops, flats, and slopes on West and Middle Anacapa.

WA, 9 May 1963, Blakley 5744 (CAS, SBBG); MA, 22 Apr 1986, Junak MA-92 (CAS, SBBG).

RHAMnACEAE

(Buckthorn Family)

Ceanothus megacarpus Nutt. var. insularis (Eastw.) Munz island big-pod ceanothus Occasional; canyons and slopes on West Anacapa. ENDEMIC to San Miguel (not seen there since 1886), Santa Rosa, Santa Cruz, Anacapa, Santa Catalina, and San Clemente islands.

WA, 9 May 1963, Blakley 5761 (RSA, SBBG)

Ceanothus megacarpus Nutt. var. megacarpus bigpod ceanothus Rare; canyon bottoms and slopes on West Anacapa.

WA, 9 May 1963, Blakley 5765a.

$$
\begin{gathered}
\text { RosaCEAE } \\
\text { (Rose Family) }
\end{gathered}
$$

Heteromeles arbutifolia (Lindl.) M. Roem. toyon Scarce; canyons on north side of West Anacapa. WA, 18 May 1961, Blakley 4380 (CAS, RSA, SBBG, US).

Prunus ilicifolia (Hook. \& Arn.) D. Dietr. subsp. lyonii (Eastw.) P.H. Raven island cherry or catalina cherry Occasional; canyons on north side of West Anacapa.

WA, 28 Mar 1965, Philbrick B65-536 (JEPS, SBBG).

$$
\begin{gathered}
\text { RubIACEAE } \\
\text { (Madder Family) }
\end{gathered}
$$

Galium angustifolium Nutt. subsp. foliosum (Hilend \& J.T. Howell) Dempster \& Stebbins narrowleaved bedstraw Common; canyons and northfacing slopes on West Anacapa and northern edge of terrace on Middle Anacapa. ENDEMIC to Santa Rosa, Santa Cruz, and Anacapa islands.

WA, 18 May 1961, Blakley 4328 (RSA, SBBG); MA, 1 Nov 1988, Junak MA-203 (RSA, SBBG).

Galium aparine L. goose-grass or cleavers Occasional; flats and north-facing slopes on all islets.

WA, 1 Apr 1962, Blakley 4988; MA, 20 Mar 1996, Junak MA-323 (RSA, SBBG); EA, 25 Apr 1959, Blakley 2775 (RSA, SBBG)

\section{SALICACEAE}

(Willow Family)

Salix lasiolepis Benth. arroyo willow Rare; known from a single collection in the middle portion of Willow Canyon on West Anacapa.

WA, 3 Oct 1978, Hochberg s.n. (SBBG 53463).

\section{SAXIFRAGACEAE \\ (Saxifrage Family)}

Heuchera maxima Greene island alum-root Scarce; canyons on north side of West Anacapa. ENDEMIC to Santa Rosa, Santa Cruz, and Anacapa islands.

WA, 17 Mar 1941, Moran 717 (DS, NY, RSA, SBBG).

SOLANACEAE

(Nightshade Family)

Lycium californicum Nutt. California box-thorn Scarce; ridgetops and terrace edges on all islets. 
WA, 13 Jan 1994, Junak WA-266; MA, 7 Sep 1964, Blakley 6527; EA, 26 Apr 1959, Blakley 2789 (CAS, JEPS, RSA, SBBG).

*Lycopersicum esculentum Mill. tomato Rare; disturbed flats and slopes on Middle and East Anacapa. First collected on northeast side of East Anacapa in August 1988 (and seen on East Anacapa by National Park Service personnel in February 1980).

MA, 1 Nov 1988, Junak MA-199 (RSA, SBBG); EA, 19 Aug 1988, Junak EA-91 (CAS, RSA, SBBG, SD).

Nicotiana clevelandii A. Gray Cleveland's tobacco Rare; ridgetops on West Anacapa.

WA, 3 Jun 1992, Junak WA-244 (RSA, SBBG).

*Solanum americanum Mill. white nightshade Rare; disturbed areas on West Anacapa, especially on extreme western end. First collected in Cherry Canyon in October 1978.

WA, 3 Oct 1978, Junak et al. WA-18.

Solanum douglasii Dunal Douglas' nightshade Rare; flats and slopes on all islets.

WA, 4 Apr 1996, Junak WA-413 (RSA, SBBG, SD); MA, 23 Nov 1986, Junak MA-217; EA, 12 May 1984, Pritchett 102.

\section{TAMARICACEAE}

(Tamarisk Family)

*Tamarix parviflora DC. small-flowered tamarisk Rare; near buildings on East Anacapa. First collected there in April 1959.

EA, 26 Apr 1959, Blakley 2786 (CAS, RSA, SBBG).

\section{URTICACEAE}

(Nettle Family)

Parietaria hespera Hinton var. californica Hinton western pellitory Rare; slopes, mostly northfacing, on all islets.

WA, 11 May 1963, Blakley 5798 (CAS, JEPS, RSA, SBBG); MA, 2 Apr 1998, Junak MA-358 (SBBG, UCR); EA, 27 Feb 1980, Junak \& Philbrick EA-49.

Urtica dioica L. subsp. holosericea (Nutt.) Thorne giant creek nettle or hoary nettle Rare; known from a single collection from "seepage cliff" at Spring Cave on West Anacapa. Not seen recently. WA, 20 August 1940, Dunkle 7648 (DS, RSA, SBBG).

*Urtica urens L. dwarf nettle Rare; known from a single collection below lighthouse on East Anacapa in February 2014, on a northeast-facing slope disturbed by seabirds.

EA, Feb 2014, Echols \& Chaney SC506.

\section{MONOCOTS \\ JUNCACEAE \\ (Rush Family)}

Juncus bufonius L. toad rush Rare; edge of vernal pool in Cherry Canyon on West Anacapa and on disturbed flats near campground on East Anacapa.

WA, 9 May 1963, Blakley 5770 (RSA, SBBG, SD); EA, 6 Apr 1993, Junak EA-101a.

\section{LILIACEAE \\ (Lily Family)}

Lilium humboldtii Duch. subsp. ocellatum (Kellogg) Thorne Humboldt lily Rare; known only from northeast-facing slope in Oak Canyon on West Anacapa.

WA, 9 May 1985, Junak et al. WA-222.

$$
\begin{aligned}
& \text { MELANTHIACEAE } \\
& \text { (False-Hellebore Family) }
\end{aligned}
$$

Toxicoscordion fremontii (Torr.) Rydb. [Zigadenus $f$. (Torr.) S. Watson] chaparral zygadene or death camas Scarce; flats and slopes on all islets.

WA, 28 Mar 1979, Junak \& Hochberg WA-57 (JEPS, SBBG); MA, 27 Mar 1965, Philbrick B67-47; EA, 18 Mar 1996, Junak EA-151 (RSA, SBBG).

$$
\begin{gathered}
\text { PoACEAE } \\
\text { (Grass Family) }
\end{gathered}
$$

*Agrostis stolonifera L. creeping bent Rare; known only from Spring Cave on north side of West Anacapa. First collected there in August 1940.

WA, 21 Aug 1940, Dunkle 7650 (DS, RSA, SBBG, $\mathrm{SD})$.

*Avena barbata Link. slender wild oat Common; flats and slopes on all islets. First collected on island (islet not specified) in June 1930.

A, 15 Jun 1930, Hoffmann s.n. (SBBG 77138); WA, 1 Apr 1962, Blakley 4986; MA, 9 May 1963, Piehl 63-192; EA, 26 Apr 1959, Blakley 2818 (CAS, OBI, RSA, SBBG, SD, UCD).

*Avena fatua L. wild oat Common; flats and slopes on all islets. First collected on island (islet not specified) in April 1939.

A, 16 Apr 1939, Bond 338; WA, 30 Mar 1995, Junak WA-369; MA, 27 Mar 1965, Philbrick B65-464a; EA, 28 Apr 1993, Junak EA-125.

*Briza minor L. small quaking grass Known from a single collection at campground on East Anacapa in June 2000.

EA, 23 Jun 2000, Junak \& Burgess EA-172.

Bromus arizonicus (Shear) Stebbins Arizona brome Scarce; flats and slopes on West and Middle Anacapa. Presence and abundance on the island varies significantly from year to year.

WA, 6 May 1980, Junak et al. WA-116; MA, 2 Apr 1998, Junak MA-348.

Bromus berteroanus Colla [B. trinii Desv. var. $t$.] Chilean chess Rare; known from a single collection on "mesa" (exact location and islet not specified) in March 1928. Not seen recently.

A, 11 Mar 1928, Hoffmann s.n. (SBBG 80252). 
Bromus carinatus Hook. \& Arn. var. carinatus California brome Scarce; north-facing slopes on West Anacapa.

WA, 9 May 1963, Blakley 5758 (CAS, SBBG).

Bromus carinatus Hook. \& Arn. var. marginatus (Steud.) Barkworth \& Anderton mountain brome Rare; flats on Middle Anacapa.

MA, 22 Apr 1986, Junak MA-88.

*Bromus catharticus Vahl var. elatus (E. Desv.) Planchuelo [B. stamineus E. Desv.] chilean brome Rare; flats and slopes on one of the islets. First collected on "mesa" (exact location and islet not specified) in March 1928. Not seen recently.

A, 11 Mar 1928, Hoffmann s.n. (SBBG 80211).

*Bromus diandrus Roth ripgut brome Common; flats and slopes on all islets. First collected on island (exact location and islet not specified) in April 1939.

A, 16 Apr 1939, Bond 329; WA, 18 May 1961, Blakley 4362 (SBBG, SD); MA, 9 May 1963, Piehl 63-233; EA, 24 Apr 1959, Blakley 2757 (CAS, SBBG, UCD).

*Bromus hordeaceus L. [B. mollis L., misapplied] soft chess Common; flats and slopes on all islets. First collected on "mesa" (exact location and islet not specified) in March 1928.

A, 11 Mar 1928, Hoffmann s.n. (SBBG 80105); WA, 18 May 1961, Blakley 4366; MA, 31 Mar 1962, Blakley 4906a; EA, 24 Apr 1959, Blakley 2744 (CAS, SBBG, UCR).

*Bromus madritensis L. subsp. rubens (L.) Husn. red brome Common; flats and slopes on all islets. First collected on "mesa" (exact location and islet not specified) in March 1928.

A, 11 Mar 1928, Hoffmann s.n. (SBBG 80257); WA, 18 May 1961, Blakley 4345; MA, 31 Mar 1962, Blakley 4903 (RSA, SBBG); EA, 26 Oct 1979, Junak \& Philbrick EA-25.

Bromus maritimus (Piper) Hitchc. maritime brome Occasional; flats and rocky slopes, especially north-facing, on West and Middle Anacapa.

WA, 3 Jun 1992, Junak WA-243 (RSA, SBBG, UCR); MA, 29 Apr 1986, Junak MA-115

Bromus sitchensis Trin. Sitka brome or Alaska brome Rare; flats and slopes on West and Middle Anacapa.

WA, 5 Apr 1962, Smith 6657; EA, 5 Aug 1974, Philbrick B74-140.

*Cynodon dactylon (L.) Pers. Bermuda grass Rare; gentle north-facing slope in seagull nesting area on Middle Anacapa. First collected at east end of Middle Anacapa in September 1964.

MA, 7 Sep 1964, Blakley 6530; MA, 22 Oct 1991, Junak MA-240 (CAS, RSA, SBBG, SD, UC).

Distichlis spicata (L.) Greene saltgrass Occasional; flats and slopes, especially along north edge of terraces, on all islets.
WA, 11 May 1963, Piehl 63-276; MA, 9 May 1963, Piehl 63-196; EA, 26 Apr 1959, Blakley 2787.

Elymus condensatus J.Presl [Leymus c. (J. Presl) Love] giant wild-rye Occasional; flats and slopes, especially along north edge of terraces, on all islets.

WA, 15 Nov 1969, Blakley 7015 (JEPS, SBBG); MA, 22 Oct 1991, Junak MA-243 (RSA, SBBG, SD); EA, 25 Apr 1959, Blakley 2773 (CAS, SBBG).

Elymus $\times$ gouldii J.P. Sm. \& Columbus Rare; disturbed flats and slopes on Middle Anacapa. This taxon is a sterile hybrid between Elymus triticoides and E. condensatus.

MA, 9 May 1963, Piehl 63-221.

Elymus triticoides Buckley [Leymus $t$. (Buckley) Pilg.] beardless wild-rye Scarce; flats and slopes, especially along north edge of terraces, on West and Middle Anacapa.

WA, 30 Aug 1990, Junak WA-238 (RSA, SBBG); MA, 22 Oct 1991, Junak MA-239 (CAS, RSA, SBBG).

*Festuca bromoides L. [Vulpia b. (L.) Gray] brome fescue Occasional; flats and slopes on all islets. First collected on island (exact location and islet not specified) in March 1929.

A, 16 Mar 1929, Hoffmann s.n. (CAS 168488; SBBG 79937); WA, 18 Mar 1967, Philbrick \& Ricker B67-9; MA, 31 Mar 1962, Blakley 4906; EA, 26 Apr 1959, Blakley 2809 (CAS, JEPS, RSA, SBBG).

Festuca microstachys Nutt. [Vulpia m. var. pauciflora (Beal) Lonard \& Gould] pacific fescue Rare; ridgetops on West Anacapa. WA, 9 Apr 1998, Junak WA-450 (CAS, RSA, SBBG).

*Festuca myuros L. [Vulpia m. (L.) C.C. Gmel. var. hirsuta Hack] foxtail fescue Scarce; flats and slopes on all islets. First collected on "mesa" (exact location and islet not specified) in March 1928.

A, 11 Mar 1928, Hoffmann s.n. (SBBG 80236); WA, 6 May 1980, Junak et al. WA-117; MA, 31 Mar 1962, Blakley 4917 (RSA, SBBG); EA, 27 Feb 1980, Junak b Philbrick EA-51.

Festuca octoflora Walter [Vulpia o. (Walter) Rydb. var. hirtella (Piper) Henrard] six-weeks fescue Rare; flats and ridgetops on all islets.

WA, 19 Mar 1967, Philbrick \& Ricker B67-30; MA, 5 Apr 1995, Junak MA-302 (RSA, SBBG); EA, 4 Apr 1995, Junak EA-137.

Hordeum brachyantherum Nevski subsp. californicum (Covas \& Stebbins) Bothmer et al. california barley or meadow barley Occasional; flats and slopes on all islets.

WA, 10 Apr 1998, Junak WA-453 (CAS, RSA, SBBG); MA, 9 May 1963, Piehl 63-198 (RSA, SBBG); EA, 26 Apr 1959, Blakley 2821.

Hordeum intercedens Nevski vernal barley or little barley Scarce; low spots on terraces and along trails on all islets. 
WA, 29 Mar 1994, Junak WA-280; MA, 1 Apr 1994, Junak MA-290; EA, 6 Apr 1993, Junak EA-103 (CAS, RSA, SBBG, SD).

*Hordeum murinum L. subsp. glaucum (Steud.) Tzvelev smooth barley or foxtail Common; disturbed flats and slopes on all islets. First collected on "mesa" on island (exact location and islet not specified) in March 1928.

A, 11 Mar 1928, Hoffmann s.n. (SBBG 79934); WA, 9 Mar 1967, Philbrick \& Ricker B67-37; MA, 7 Apr 1968, Philbrick \& Osborne B68-158; EA, 5 Aug 1974, Philbrick B74-145.

*Hordeum murinum L. subsp. leporinum (Link) Arcang. hare barley or foxtail Common; disturbed flats and slopes on all islets. First collected on "poor soil" on island (exact location and islet not specified) in March 1929.

A, 16 Mar 1929, Hoffmann s.n. (SBBG 79933); WA, 19 Mar 1967, Philbrick \& Ricker B67-36; MA, 9 May 1963, Piehl 63-224; EA, 24 Apr 1959, Blakley 2748.

*Lamarckia aurea (L.) Moench. goldentop Common; flats and slopes on all islets. First collected "between the boat landing and light station housing area" on East Anacapa in April 1959.

WA, 8 May 1980, Junak WA-164 (RSA, SBBG); MA, 31 Mar 1962, Blakley 4881 (JEPS, OBI, SBBG, SD); EA, 24 Apr 1959, Blakley 2752 (CAS, SBBG, UCD).

Melica imperfecta Trin. Coast Range melic Occasional; flats and slopes on all islets.

WA, 30 Mar 1995, Junak WA-391 (RSA, SBBG); MA, 22 Apr 1986, Junak MA-112 (CAS, SBBG); EA, 18 Mar 1996, Junak EA-150 (RSA, SBBG).

Muhlenbergia microsperma (DC.) Kunth littleseed muhly Scarce; slopes, especially south-facing, on West and Middle Anacapa.

WA, 29 Mar 1995, Junak WA-342 (RSA, SBBG); MA, 27 Mar 1979, Junak \& Philbrick MA-14.

*Parapholis incurva (L.) C.E. Hubb. sickle grass Rare; flats on East Anacapa. First collected along trail, just east of campground, on East Anacapa in May 1982.

EA, 21 May 1982, Junak EA-73; EA, 9 Jul 1998, Junak EA-161.

*Pennisetum clandestinum Chiov. kikuyu grass Rare; flats and north-facing slopes disturbed by nesting seagulls on Middle and East Anacapa. First collected just downslope from lighthouse on East Anacapa in August 1988.

MA, 23 Nov 1988, Junak MA-214 (CAS, RSA, SBBG); EA, 19 Aug 1988, Junak EA-89 (CAS, RSA, SBBG, SD, UC).

*Phalaris minor Retz. little-seeded canary grass Scarce; flats and slopes on all islets. First collected on island (exact location and islet not specified) in June 1930.

A, 16 Jun 1930, Hoffmann s.n. (SBBG 77173); WA, 11 May 1963, Piehl 63-269; MA, 9 May 1963, Blakley
5738 (CAS, RSA, SBBG, UCR); EA, 6 Apr 1993, Junak EA-104.

*Phalaris paradoxa L. hood canary grass Scarce; disturbed flats on East Anacapa. First collected along trail between water tank building and water catchment pad in June 1997.

EA, 17 Jun 1997, Junak \& Kirkland EA-158.

*Poa annua L. annual bluegrass Rare; disturbed flats and slopes on all islets. First collected along trail just west of ranger residence on East Anacapa in March 1979.

WA, 9 Apr 1998, Junak WA-444 (RSA, SBBG); MA, 20 Mar 1996, Junak MA-327; EA, 30 Mar 1979, Philbrick \& Hochberg B79-8.

Poa secunda J. Presl subsp. secunda [P. scabrella (Thurb.) Vasey] one-sided bluegrass Occasional; flats and north-facing slopes on all islets.

WA, 6 Apr 1968, Philbrick \& Osborne B68-134 (RSA, SBBG); MA, 31 Mar 1962, Blakley 4913 (CAS, RSA, SBBG); EA, 27 Feb 1980, Junak \& Philbrick EA-44 (RSA, SBBG).

*Polypogon monspeliensis (L.) Desf. rabbitfoot grass Rare; low spots on flats on Middle and East Anacapa. First collected near buildings on East Anacapa in August 1974.

MA, 3 Jun 1978, Philbrick \& Hochberg B78-341; EA, 5 Aug 1974, Philbrick B74-149.

*Sorghum bicolor (L.) Moench. sorghum Rare; known from a single collection "around water faucet in front of bunkhouse" on East Anacapa in July 1984.

EA, 27 Jul 1984, Drost 386.

Stipa cernua Stebbins \& Love [Nassella c. (Stebbins \& Love) Barkworth] nodding needle grass Scarce; ridgetops and slopes on West and East Anacapa. WA, 3 Jun 1992, Junak WA-258; EA, 28 Apr 1993, Junak EA-128.

Stipa diegoensis Swallen [Achnatherum d. (Swallen) Barkworth] San Diego needle grass Occasional; flats and slopes on all islets. This taxon reaches its northern limits on the Northern Channel Islands (Ferren et al. 1986).

WA, 3 Jun 1992, Junak WA-248 (RSA, SBBG, SD); MA, 29 Apr 1986, Junak MA-133 (RSA, SBBG, SD); EA, 6 Apr 1993, Junak EA-105 (RSA, SBBG).

Stipa lepida Hitchc. [Nassella l. (Hitchc.) Barkworth] foothill needle grass Rare; slopes on north side of West Anacapa.

WA, 18 May 1961, Blakley 4339.

*Stipa miliacea (L.) Hoover var. miliacea [Piptatherum m. (L.) Coss. subsp. m.] smilo grass or rice grass Rare; disturbed flats and slopes, especially on north side, of Middle Anacapa. First collected in seagull nesting area above Lavatera Cove in November 1988.

MA, 22 Nov 1988, Junak MA-211 (RSA, SBBG). 
Stipa pulchra Hitchc. [Nassella p. (Hitchc.) Barkworth] purple needle grass Occasional; flats and slopes on all islets.

WA, 3 Jun 1992, Junak WA-254 (RSA, SBBG); MA, 31 Mar 1994, Junak MA-256; EA, 22 Apr 1970, Benedict s.n. (SBBG 43151).

\section{THEMIDACEAE}

(Brodiaea Family)

Dichelostemma capitatum (Benth.) Alph. Wood subsp. capitatum blue dicks Common; flats on all islets. Apparent abundance varies significantly from year to year

WA, 30 Mar 1995, Junak WA-364 (RSA, SBBG, SD); MA, 31 Mar 1962, Blakley 4919 (RSA, SBBG); EA, 26 Apr 1959, Blakley 2796 (JEPS, RSA, SBBG, SD, UCR).

\section{ZOSTERACEAE (Eel-Grass Family)}

Phyllospadix scouleri Hook. surf-grass Occasional; known from shallow water around perimeter of Middle Anacapa. Distribution needs further study.

MA; 29 Aug 1940, Dunkle 7673 (RSA, SBBG).

Phyllospadix torreyi S.Watson surf-grass Occasional; known from shallow water at and near Frenchy's Cove on West Anacapa. Distribution needs further study.

WA, 28 May 1963, Blakley 5927.

Zostera marina L. eel-grass Scarce; waters near Frenchy's Cove on West Anacapa, north side of Middle Anacapa, and Landing Cove on East Anacapa. Distribution needs further study.

WA, 24 May 1969, Bleck s.n. (RSA 684212); MA, 2 Jun 1978, Philbrick of Hochberg B78-296 (JEPS, SBBG); EA, 23 Apr 1982, Philbrick s.n. (SBBG).

\section{ACKNOWLEDGMENTS}

The authors thank the superintendents, boat captains, and staff of Channel Islands National Park, especially Diane Brooks, Keith Duran, Dave Stoltz, Dwight Willey, Charlie Bird, Pat Given, Bill Ehorn, and Tim Glass for exceptional logistical support on our trips to Anacapa Island. We thank Island Packers, NOAA, the National Marine Sanctuary, and Peter Howorth for additional boat transportation.

We greatly appreciate the support of the Santa Barbara Botanic Garden and thank the curators and staff of herbaria at the California Academy of Sciences, Jepson Herbarium, New York Botanic Garden, Rancho Santa Ana Botanic Garden, San Diego Museum of Natural History, Smithsonian Institution, and Uni- versity of California at Berkeley for access to critical specimens and for many favors.

Sarah Chaney, Dan Richards, Dirk Rodriguez, Bill Halvorson, Karen Danielson, Trudy Ingram, Ken Owen, Ronie Fowler Clark, Cathy Schwemm, Frank Ugolini, Frank Gress, and Bill Erickson helped in many ways, as did Marla Daily, Clif Smith, Paul Collins, John Knapp, Betsy Lape, and Dewey Livingston.

We also thank Keri Kirkland, Dave Rosen, Mary Carroll, Don Ricker, Barry Osborne, Ed Philbrick, Dave Kershaw, Gail Meadows Milliken, Sally Isaacson, Stan Davis, Aaron Barton, Aaron Echols, Steve Williams, Steven Timbrook, Caroline Kuizenga, Rick Burgess, George Leone, Heather Leone, Eric Hochberg, and John Amick for help in the field or for sharing their collection data.

Two anonymous reviewers provided valuable information and corrections to the draft of this manuscript. Their comments were greatly appreciated!

\section{Literature Cited}

Abrams, L. 1940-1951. Illustrated flora of the Pacific states. Volumes 1-3. Stanford University Press, Stanford, CA.

Abrams, L., AND R. FerRis. 1960. Illustrated flora of the Pacific states. Volume 4. Stanford University Press, Stanford, CA.

Baldwin, B., D. Goldman, D. Keil, R. Patterson, T. Rosatti, And D. Wilken, Editors. 2012. The Jepson manual: vascular plants of California. 2nd edition. University of California Press, Berkeley, CA.

BANKS, R. 1966. Terrestrial vertebrates of Anacapa Island, California. Transactions of the San Diego Society of Natural History 14:173-188.

Camp, C. 1963. Lorenzo Gordin Yates (1837-1909). Journal of the Society for the Bibliography of Natural History 4:178-193.

DunkLE, M. 1950. Plant ecology of the Channel Islands of California. Publications of the Allan Hancock Pacific Expeditions 13:247-386.

GLEAsON, J. 1950. Islands of California: their history, romance, and physical characteristics. Sea Publications, Los Angeles, CA.

Holmgren, P., N. Holmgren, and L. Barnett. 1990. Index Herbariorum, Part 1: The herbaria of the world. 8th edition. Regnum Vegetabile 120:1-693.

Johnson, D. 1979. Geology, soils, and erosion. Pages 3.1-3.79 in D. Power, editor, Natural resources study of the Channel Islands National Monument, California. Santa Barbara Museum of Natural History, Santa Barbara, CA

JunAK, S. 2008. A flora of San Nicolas Island. Santa Barbara Botanic Garden, Santa Barbara, CA.

Junak, S., T. Ayers, R. Scott, D. Wilken, and D. Young. 1995. A flora of Santa Cruz Island. Santa Barbara Botanic Garden and California Native Plant Society, Santa Barbara and Sacramento, CA. 
Junak, S., S. Chaney, R. Philbrick, and R. Clakk. 1997. A checklist of the vascular plants of Channel Islands National Park. 2nd edition. Southwest Parks and Monuments Association, Tucson, AZ.

Livingston, D. 2006. Historic resource study: a history of the islands within Channel Islands National Park. Section 5: Anacapa Island. Technical Report submitted to Channel Islands National Park, Ventura, CA.

McEachern, K., T. Atwater, P. Collins, K. Faulkner, AND D. Richards. 2016. Managed island ecosystems. Pages 755-778 in H. Mooney and E. Zavaleta, editors, Ecosystems of California. University of California Press, Berkeley, CA.

Roberts, L. 1979. Historic resource study: Channel Islands National Monument and San Miguel Island, California. Chambers Consultants and Planners, Orange County, CA.

Roberts, L. 1983. Anacapa Island. McNally and Loftin Publishers, Santa Barbara, CA.

SCHOLL, D. 1960. Relationship of the insular shelf sediments to the sedimentary environments and geology of Anacapa Island, California. Journal of Sedimentary Petrology 30:123-139.

Smith, C. 1998. A flora of the Santa Barbara region, California. 2nd edition. Santa Barbara Botanic Garden and Capra Press, Santa Barbara, CA.
Vedder, J. 1976. Precursors and evolution of the name California Continental Borderland. Pages 6-11 in D. Howell, editor, Aspects of the geologic history of the California Continental Borderland. Miscellaneous Publication 24. American Association of Petroleum Geologists, Pacific Section, Tulsa, OK.

Weissman, D. and D. Rentz. 1977. Rainfall data for the California Channel Islands and adjacent mainland. Unpublished manuscript.

Western Regional Climate Center. 2017. Anacapa Island California monthly summary time series. [Accessed 16 August 2017]. https://wrcc.dri.edu/ channel_isl/index.html.

YATES, L. 1890. Notes on the geology and scenery of the islands forming the southerly line of the Santa Barbara Channel. American Geologist 14:43-52.

Received 23 March 2017

Revised 20 October 2017

Accepted 26 October 2017

Published online 15 February 2018 